\title{
MULTISCALE MODELLING OF CHEMICAL DEGRADATION MECHANISMS IN POROUS MEDIA WITH EVOLVING MICROSTRUCTURE*
}

\author{
MALTE A. PETER ${ }^{\dagger}$ AND M. BÖHM ${ }^{\ddagger}$
}

\begin{abstract}
A prototypical reaction-diffusion system in a porous medium is considered, whose microstructure undergoes an evolution with respect to time. Employing the recently developed method of homogenization in domains with evolving microstructure, the limit problems are obtained. Besides the time-evolution aspect, attention is paid to the scaling of the material parameters with powers of the homogenization parameter arising from a nondimensionalization. The paper has two main focuses: One focus is numerical experiments for prototypical simplified problems, which qualitatively confirm the appropriateness of the scalings obtained from a nondimensionalization and show the influence of the evolution of the microstructure on the model output. The second main focus is the real-world problem concrete carbonation, which is considered as a typical application of the presented approach. Comparison of experimental data for concrete carbonation with the simulation results confirms the validity of the modelling approach.
\end{abstract}

Key words. homogenization, evolution of the microstructure, reaction-diffusion, concrete carbonation

AMS subject classifications. 35B27, 35K57, 74F25, 92E20

DOI. $10.1137 / 070706410$

1. Introduction. Reaction-diffusion processes in media with microstructure occur in a variety of different applications. Many classical results have been obtained in the context of porous media (cf. $[3,16,13]$, e.g.), but there are also more recent results in other directions, such as the calcium balance in biological cells [11]. An important new aspect in reaction-diffusion processes in media with microstructure is accounting for an evolution of the different parts of the medium. Some approaches in this direction have been put forward in the context of phase transitions in alloys [1] or liquid-solid phase transitions of binary mixtures [10, 9], for example. Recently, the method of homogenization in domains with evolving microstructure [25] was introduced, which allows the transformation of the problem with evolving microstructure to a form suitable for a periodic-homogenization analysis. The main aspects of this paper are to show how this method can be used for a prototypical reaction-diffusion process in a porous medium with evolving microstructure and, as an illustrating (and in some way prototypical) example, its application to a particular real-world problem, namely concrete carbonation.

We consider the prototypical problem of reaction, diffusion, and interfacial exchange in a porous medium $\Omega$ made up of three distinct parts, that is, the pore-air space, $\Omega^{\mathrm{a}}(t)$, the pore-water space, $\Omega^{\mathrm{w}}(t)$, and the space occupied by solid matrix,

${ }^{*}$ Received by the editors October 25, 2007; accepted for publication (in revised form) February 11, 2009; published electronically April 24, 2009. This work was supported by Special Priority Program 1122 "Prediction of the course of physico-chemical damage processes involving mineral materials" of the German Research Foundation, which provided insights into concrete carbonation.

http://www.siam.org/journals/mms/7-4/70641.html

${ }^{\dagger}$ Centre for Industrial Mathematics, FB 3, University of Bremen, P.O. Box 330 440, 28334 Bremen, Germany. Current address: Institute of Mathematics, University of Augsburg, 86135 Augsburg, Germany (malte.peter@math.uni-augsburg.de). This author's research was supported by the German National Academic Foundation.

${ }^{\ddagger}$ Centre for Industrial Mathematics, FB 3, University of Bremen, P.O. Box 330 440, 28334 Bremen, Germany (mbohm@math.uni-bremen.de). 
$\Omega^{\mathrm{s}}(t)$, in the time interval $S=(0, T)$, where $T>0$. The evolution of the parts of $\Omega$ is induced by a reaction. One substance diffuses through the pore air and dissolves into the pore water where it reacts with a second substance. In the reaction, the second substance is consumed or produced. Denoting the reacting substances by A and B, respectively, the reaction may be expressed by

$$
n_{\mathrm{A}} \mathrm{A}+n_{\mathrm{B}}^{\mathrm{con}} \mathrm{B} \longrightarrow n_{\mathrm{B}}^{\mathrm{pro}} \mathrm{B}+n_{\mathrm{C}} \mathrm{C},
$$

where $\mathrm{C}$ is a reaction product and the natural numbers $n_{i}, i \in\{\mathrm{A}, \mathrm{B}, \mathrm{C}\}$, are stoichiometric factors where the superscripts con and pro stand for consumed and produced, respectively. Other products may be formed but are not further considered in this work since they do not add any conceptually new ideas. The only assumption on the stoichiometric factors is that they are nonnegative.

An important application of the process under consideration is concrete carbonation, which motivates the consideration of this particular problem. Concrete carbonation is described in more detail in section 1.2. The modelling ideas presented here are also applicable to reaction-diffusion scenarios in other materials with or without evolving microstructure, biological cells, for example, where the evolution of the subdomains may be due to a movement of the endoplasmic reticulum, or to heat-transfer and fluid-flow problems.

The reaction-diffusion problem is given in a domain, whose microstructure undergoes an evolution with respect to time. In order to use periodic-homogenization techniques (cf. $[4,33]$, e.g.), the method of homogenization in domains with evolving microstructure [25] is used for coping with the changing microstructure. This method is based on transforming the problem from the current configuration to an associated periodic reference configuration and to homogenize the resulting transformed problem. In particular, the method allows for nonperiodic evolving domains.

It is well known that scaling of the material parameters with powers of the homogenization parameter has a strong influence on the type of limit problem (cf. [28, 22, 21], e.g.). It is therefore of great importance to use the correct scaling in order to obtain useful macroscopic models. We introduce a nondimensionalization in order to obtain problem-dependent scalings, which lead to process-adapted models in the homogenization limit. The particular choice of scaling then depends on the characteristic lengths of the medium as well as the diffusivities. The limit problems are given for general scalings.

The objective of the simulation results presented in the second part of the paper is twofold: First, the appropriateness of the limit problems depending on the scalings and the influence of the evolution of the microstructure is shown by simulations for prototypical simplified versions of the general problem. In particular, it turns out that correct scalings (in terms of the proposed nondimensionalization) lead to useful, process-adapted limit models while wrong scalings give inappropriate limit models. Second, the general results are applied to the real-world problem concrete carbonation for which the evolution is coupled to the reaction-diffusion system. The simulation results show a good match with experimental data.

1.1. The prototypical reaction-diffusion problem. We denote the mass concentrations of substances $\mathrm{A}$ and $\mathrm{B}$ by $u=u(x, t)$ and $v=v(x, t)$, respectively, where $x$ and $t$ are the space and time variables, respectively, and we distinguish between the concentration in the pore air and the pore water by superscripts a and $\mathrm{w}$, respectively. Note that $v$ is assumed to occur in the pore water only. Hence, there is no $v^{\mathrm{a}}$. 
There are three different interfaces in the pores: the air-water interface, $\Gamma^{\mathrm{aw}}(t)$, the water-solid interface, $\Gamma^{\mathrm{ws}}(t)$, and the air-solid interface $\Gamma^{\mathrm{as}}(t)$. For ease of notation, we write $\Gamma^{\mathrm{w}}(t)=\Gamma^{\mathrm{aw}}(t) \cup \Gamma^{\mathrm{ws}}(t)$.

The consumption rates of constituents $\mathrm{A}$ and $\mathrm{B}$ associated with the reaction (1.1) are assumed to be of the form

$$
\eta^{\mathrm{u}}=R^{\mathrm{u}} r_{\mathrm{u}}\left(u^{\mathrm{w}}\right) r_{\mathrm{v}}\left(v^{\mathrm{w}}\right) \quad \text { and } \quad \eta^{\mathrm{v}}=R^{\mathrm{v}} r_{\mathrm{u}}\left(u^{\mathrm{w}}\right) r_{\mathrm{v}}\left(v^{\mathrm{w}}\right),
$$

respectively, where $R^{\mathrm{u}}, R^{\mathrm{v}}: \Omega^{\mathrm{w}} \times S \rightarrow \mathbb{R}$ are bounded and $r_{\mathrm{u}}, r_{\mathrm{v}}: \mathbb{R} \rightarrow \mathbb{R}$ are locally Lipschitz continuous. Moreover, $r^{\mathrm{u}}$ is assumed nonnegative while $r^{\mathrm{v}}$ is assumed either nonnegative or nonpositive depending on $n_{\mathrm{B}}^{\text {con }}-n_{\mathrm{B}}^{\text {pro }}$ being negative or positive. Typically, the concentration-dependent part of the production rate, $f^{\mathrm{r}}=r_{\mathrm{u}} r_{\mathrm{v}}$, is of the form

$$
f^{\mathrm{r}}=\left(u^{\mathrm{w}}\right)^{p}\left(v^{\mathrm{w}}\right)^{q}
$$

with $p, q \in\{s \in \mathbb{R} \mid s=0$ or $s \geq 1\}$ and the factors $R^{i}, i \in\{\mathrm{u}, \mathrm{v}\}$, incorporate the molar weights of the respective substances and the (possibly time- and spacedependent) reaction-rate constant.

The reaction-diffusion problem under consideration is then given as follows. The mass balances of the species are

$$
\begin{aligned}
\partial_{t} u^{\mathrm{a}}(x, t)-\nabla \cdot\left(D^{1} \nabla u^{\mathrm{a}}-w^{\mathrm{a}} u^{\mathrm{a}}\right) & =0, & & x \in \Omega^{\mathrm{a}}(t), t \in S, \\
\partial_{t} u^{\mathrm{w}}(x, t)-\nabla \cdot\left(D^{2} \nabla u^{\mathrm{w}}-w^{\mathrm{w}} u^{\mathrm{w}}\right) & =-R^{\mathrm{u}} f^{\mathrm{r},} & & x \in \Omega^{\mathrm{w}}(t), t \in S, \\
\partial_{t} v^{\mathrm{w}}(x, t)-\nabla \cdot\left(D^{3} \nabla v^{\mathrm{w}}-w^{\mathrm{w}} v^{\mathrm{w}}\right) & =+R^{\mathrm{v}} f^{\mathrm{r}}, & & x \in \Omega^{\mathrm{w}}(t), t \in S,
\end{aligned}
$$

where $D^{i}=D^{i}(x, t), i \in\{1,2,3\}$, are the corresponding diffusivities and $w^{i}, i \in$ $\{\mathrm{a}, \mathrm{w}\}$, are the velocities of the deformation of the respective domains. The boundary conditions at the internal boundaries of the porous medium are given by

$$
\begin{aligned}
-\left(D^{1} \nabla u^{\mathrm{a}}\right) \cdot \nu^{\mathrm{a}}= & \left(D^{2} \nabla u^{\mathrm{w}}\right) \cdot \nu^{\mathrm{w}}=C^{\mathrm{ex}}\left(C^{\mathrm{H}} u^{\mathrm{a}}-u^{\mathrm{w}}\right), & & x \in \Gamma^{\mathrm{aw}}(t), t \in S, \\
& -\left(D^{1} \nabla u^{\mathrm{a}}\right) \cdot \nu^{\mathrm{a}}=0, & & x \in \Gamma^{\mathrm{as}}(t), t \in S, \\
& -\left(D^{2} \nabla u^{\mathrm{w}}\right) \cdot \nu^{\mathrm{w}}=0, & & x \in \Gamma^{\mathrm{ws}}(t), t \in S, \\
& -\left(D^{3} \nabla v^{\mathrm{w}}\right) \cdot \nu^{\mathrm{w}}=0, & & x \in \Gamma^{\mathrm{w}}(t), t \in S,
\end{aligned}
$$

where $\nu^{i}$ denotes the outer normal on $\partial \Omega^{i}(t), i \in\{\mathrm{a}, \mathrm{w}\}$, the positive Henry constant $C^{\mathrm{H}}$ describes the ratio of $u^{\mathrm{w}}$ and $u^{\mathrm{a}}$ in equilibrium, and $C^{\mathrm{ex}}=C^{\mathrm{ex}}(x, t)$ is related to the rate of interfacial exchange. For now, we assume no-flux conditions at the external interface $\partial \Omega$ for simplicity. The system is completed by nonnegative initial conditions $u^{\mathrm{a}}(x, 0)=u_{0}^{\mathrm{a}}(x), u^{\mathrm{w}}(x, 0)=u_{0}^{\mathrm{w}}(x)$, and $v^{\mathrm{w}}(x, 0)=v_{0}^{\mathrm{w}}(x)$.

For ease of notation, we write $\Gamma(t)=\Gamma^{\text {aw }}(t)$ and assume $\Gamma^{\text {as }}(t)=\emptyset$. This does not imply any restriction from the mathematical modelling point of view since there is no production or consumption of any type at this boundary. However, it simplifies the presentation considerably.

It is noteworthy that the particular form of the reaction-diffusion system under consideration is motivated by the real-world problem concrete carbonation, which is discussed in more detail in the next section. Nevertheless, it is a prototypical reactiondiffusion system since it captures many subprocesses often encountered in applications, that is, diffusion, reaction, and interfacial exchange of different substances in different parts of the porous medium. The methods employed and developed in the subsequent sections also apply to similar reaction-diffusion systems and are not restricted to the particular form considered here. 
1.2. A real-world application: Concrete carbonation. The problem under consideration applies to a variety of chemical degradation mechanisms in porous media, in particular to concrete carbonation. This process takes place in the pores of concrete, which are partially saturated with water that clings to the pore walls, and involves reaction, diffusion, precipitation, and dissolution. Atmospheric $\mathrm{CO}_{2}$ enters the concrete through the air-filled pores and dissolves in the pore water. There, it reacts with dissolved constituents of the cement paste, most importantly with $\mathrm{Ca}(\mathrm{OH})_{2}$. This causes a lowering of the $\mathrm{pH}$, facilitating the corrosion of the steel reinforcements and, consequently, leads to a severe reduction of the service life of the structure. The dominant carbonation reaction is usually assumed as

$$
\mathrm{CO}_{2}(a q)+\mathrm{Ca}(\mathrm{OH})_{2}(a q) \longrightarrow \mathrm{CaCO}_{3}(a q)+\mathrm{H}_{2} \mathrm{O} .
$$

The produced $\mathrm{CaCO}_{3}$ precipitates very quickly to the solid matrix. In view of the general reaction (1.1), we have $\mathrm{A}=\mathrm{CO}_{2}$ and $\mathrm{B}=\mathrm{Ca}(\mathrm{OH})_{2}$ as well as $n_{\mathrm{A}}=n_{\mathrm{B}}^{\text {con }}=1$ and $n_{\mathrm{B}}^{\mathrm{pro}}=0$. Detailed surveys on the carbonation problem were carried out, for instance, by [14], [5], and [7] and, from a more mathematical point of view, by [19].

An important feature of concrete carbonation is that the carbonation reaction causes a change of the microstructure in at least two ways. First, the reactant $\mathrm{Ca}(\mathrm{OH})_{2}$ takes up considerably less volume than the product $\mathrm{CaCO}_{3}$. This causes a permanent reduction of the pore-air volume. Second, water is produced in the carbonation reaction. This induces a (usually) temporary reduction of the pore-air volume and an increase of the volume of the reaction medium. While it seems that the latter effect is of considerable importance only in accelerated carbonation tests (as opposed to carbonation under natural atmospheric conditions) (cf. [20]), the first effect is always important because it permanently slows down the diffusion of $\mathrm{CO}_{2}(g)$ to the reaction zone and, in turn, reduces the speed of the overall carbonation process; also cf. [18]. A detailed discussion of these effects and their impact on the progress of carbonation can be found in [20]. In section 6, the application of the theoretical results to the particular problem of concrete carbonation is discussed in more detail and numerical simulations of concrete carbonation are presented.

1.3. Structure of the paper. In section 2 , system (1.4) is nondimensionalized taking into account two relevant characteristic lengths: a microscopic length and a macroscopic length. The resulting dimensionless system is put into a form suitable for a homogenization analysis in the context of homogenization in domains with evolving microstructure in section 3 , and the homogenized limit problems are given in section 4 . Qualitative simulation results for prototypical problems are presented in section 5 in order to illustrate the appropriateness and applicability of the resulting limit models for a given evolution. In section 6 , the theoretical results are applied to the particular problem of concrete carbonation where the evolution of the microstructure is coupled to the reaction-diffusion system. Comparison of the simulation results with experimental data for concrete carbonation confirms the validity of the model.

2. Nondimensionalization. In order to obtain process-adapted models in the homogenization limit, it is important to identify the characteristic microscopic and macroscopic lengths and to properly nondimensionalize the system of equations (cf. $[29,21]$, e.g.). In this section, a possible nondimensionalization of problem (1.4) is given, which is similar to that given in [28] for a very much simplified version of the problem considered here. In order to keep the presentation concise, $u^{\mathrm{a}}$ is assumed to be the fastest diffusing species. 
The following dimensionless concentrations are introduced,

$$
\tilde{u}^{\mathrm{a}}:=u^{\mathrm{a}} C^{\mathrm{H}} / u_{\mathrm{ref}}^{\mathrm{a}}, \quad \tilde{u}^{\mathrm{w}}:=u^{\mathrm{w}} / u_{\mathrm{ref}}^{\mathrm{w}}, \quad \tilde{v}^{\mathrm{w}}:=v^{\mathrm{w}} / v_{\mathrm{ref}}^{\mathrm{w}},
$$

where $u_{\text {ref }}^{\alpha}, \alpha \in\{\mathrm{a}, \mathrm{w}\}$, and $v_{\text {ref }}^{\mathrm{w}}$ are some reference concentrations representing upper bounds on the concentrations. These may be given from physical considerations or maximum estimates, for example.

Moreover, we introduce a characteristic macroscopic length $L$ (the diameter of the sample, e.g.) and a characteristic microscopic length $\ell$ (a typical pore diameter, e.g.). For each concentration $u^{\mathrm{a}}, u^{\mathrm{w}}$, and $v^{\mathrm{w}}$, we define a characteristic length associated with the diffusion of the species in the pore, $\ell_{i}^{\mathrm{D}}, i=1,2,3$, respectively, and express this as a multiple of $\ell$ in the following way: $\ell_{i}^{\mathrm{D}}=\sqrt{D_{i}} \ell$ (note the difference between the diffusivity $D^{i}$ and the number $D_{i}$ ). Letting $D_{\text {ref }}^{i}$ be the $L^{\infty}$-bounds of $D^{i}, i=1,2,3$, the characteristic diffusion times are then defined as

$$
T^{\mathrm{a}}:=\left(\ell_{1}^{\mathrm{D}}\right)^{k} L^{2-k} / D_{\mathrm{ref}}^{1}, \quad T^{\mathrm{w}}:=\left(\ell_{2}^{\mathrm{D}}\right)^{l} L^{2-l} / D_{\text {ref }}^{2}, \quad T_{\mathrm{v}}^{\mathrm{w}}:=\left(\ell_{3}^{\mathrm{D}}\right)^{j} L^{2-j} / D_{\text {ref }}^{3},
$$

where $k, l, j \in[0,2]$ are to be specified later (see section 2.1). These are the scaling exponents, the discussion of which is one of the focal points of this paper. Also, set $\varepsilon=\ell / L$.

Making use of the characteristic lengths introduced earlier, let $\tilde{x}:=x / L$ and $t^{\alpha}:=t / T^{\alpha}$ and $t_{\mathrm{v}}^{\mathrm{w}}:=t / T_{\mathrm{v}}^{\mathrm{w}}$ be the dimensionless (macroscopic) space and time variables and write $\tilde{u}^{\alpha}\left(\tilde{x}, t^{\alpha}\right):=u^{\alpha}\left(\tilde{x} L, t^{\alpha} T^{\alpha}\right), \alpha \in\{\mathrm{a}, \mathrm{w}\}$, and, analogously, for $v^{\mathrm{w}}$. Note that the corresponding time intervals are then given by $\tilde{S}^{\mathrm{a}}:=S / T^{\mathrm{a}}, \tilde{S}^{\mathrm{w}}:=S / T^{\mathrm{w}}$, and $\tilde{S}_{\mathrm{v}}^{\mathrm{w}}:=S / T_{\mathrm{v}}^{\mathrm{w}}$. Moreover, we introduce the parameter $m \in \mathbb{R}$ related to the speed of the interfacial exchange. From a physical point of view, condition (1.4d) motivates the assumption that both concentrations tend toward the equilibrium $u_{\mathrm{ref}}^{\mathrm{a}} \tilde{u}^{\mathrm{a}}=u_{\mathrm{ref}}^{\mathrm{w}} \tilde{u}^{\mathrm{w}}$. Therefore, it is reasonable to take $u_{\mathrm{ref}}^{\mathrm{w}}=u_{\mathrm{ref}}^{\mathrm{a}}=: u_{\mathrm{ref}}$, which makes the problem less technical. Notice that this simplification is not necessary, however. Introducing the dimensionless functions (where the change of arguments is shown only for $\tilde{D}^{\text {a }}$ for readability)

$$
\begin{gathered}
\tilde{D}^{\mathrm{a}}\left(\tilde{x}, t^{\mathrm{a}}\right):=\frac{D_{1}^{\frac{k}{2}}}{D_{\text {ref }}^{1}} D^{1}\left(\tilde{x} L, \tilde{t}^{\mathrm{a}} T^{\mathrm{a}}\right), \quad \tilde{D}^{\mathrm{w}}:=\frac{D_{2}^{\frac{l}{2}}}{D_{\text {ref }}^{2}} D^{2}, \quad \tilde{E}^{\mathrm{w}}:=\frac{D_{3}^{\frac{j}{2}}}{D_{\text {ref }}^{3}} D^{3}, \\
\tilde{w}^{\mathrm{a}}:=\frac{T^{\mathrm{a}}}{L} w^{\mathrm{a}}, \quad \tilde{w}^{\mathrm{w}}:=\frac{T^{\mathrm{w}}}{L} w^{\mathrm{w}}, \\
\tilde{C}_{\mathrm{ex}}^{\mathrm{a}}:=\ell^{-m} L^{m-1} T^{\mathrm{a}} C^{\mathrm{H}} C^{\mathrm{ex}}, \quad \tilde{C}_{\mathrm{ex}}^{\mathrm{w}}:=\ell^{-m} L^{m-1} T^{\mathrm{w}} C^{\mathrm{ex}}, \\
\tilde{R}^{\mathrm{u}}:=\frac{T^{\mathrm{w}}}{u_{\mathrm{ref}}} R^{\mathrm{u}}, \quad \tilde{R}^{\mathrm{v}}:=\frac{T_{\mathrm{v}}^{\mathrm{w}}}{v_{\text {ref }}} R^{\mathrm{v}}
\end{gathered}
$$

and writing $\tilde{f}^{\mathrm{r}}:=r^{\mathrm{u}}\left(\tilde{u}^{\mathrm{w}} u_{\mathrm{ref}}^{\mathrm{w}}\right) r^{\mathrm{v}}\left(\tilde{v}^{\mathrm{w}} v_{\mathrm{ref}}^{\mathrm{w}}\right)$, the dimensionless version of system (1.4) is given by

$$
\begin{array}{cc}
\partial_{t^{\mathrm{a}}} \tilde{u}^{\mathrm{a}}-\nabla \cdot\left(\varepsilon^{k} \tilde{D}^{\mathrm{a}} \nabla \tilde{u}^{\mathrm{a}}-\tilde{w}^{\mathrm{a}} \tilde{u}^{\mathrm{a}}\right)=0, & \tilde{x} \in \Omega^{\mathrm{a}}(t), \\
\partial_{t^{\mathrm{w}}} \tilde{u}^{\mathrm{w}}-\nabla \cdot\left(\varepsilon^{l} \tilde{D}^{\mathrm{w}} \nabla \tilde{u}^{\mathrm{w}}-\tilde{w}^{\mathrm{w}} \tilde{u}^{\mathrm{w}}\right)=-\tilde{R}^{\mathrm{u}} \tilde{f}^{\mathrm{r}}, & \tilde{x} \in \Omega^{\mathrm{w}}(t), \\
\partial_{t_{\mathrm{v}}^{\mathrm{w}}} \tilde{v}^{\mathrm{w}}-\nabla \cdot\left(\varepsilon^{j} \tilde{E}^{\mathrm{w}} \nabla \tilde{v}^{\mathrm{w}}-\tilde{w}^{\mathrm{w}} \tilde{v}^{\mathrm{w}}\right)=+\tilde{R}^{\mathrm{v}} \tilde{f}^{\mathrm{r}}, & \tilde{x} \in \Omega^{\mathrm{w}}(t), \\
-\varepsilon^{k} \tilde{D}^{\mathrm{a}} \nabla \tilde{u}^{\mathrm{a}} \cdot \nu^{\mathrm{a}}=\varepsilon^{m} \tilde{C}_{\mathrm{ex}}^{\mathrm{a}}\left(\tilde{u}^{\mathrm{a}}-\tilde{u}^{\mathrm{w}}\right), & \tilde{x} \in \Gamma(t), \\
-\varepsilon^{l} \tilde{D}^{\mathrm{w}} \nabla \tilde{u}^{\mathrm{w}} \cdot \nu^{\mathrm{w}}=-\varepsilon^{m} \tilde{C}_{\mathrm{ex}}^{\mathrm{w}}\left(\tilde{u}^{\mathrm{a}}-\tilde{u}^{\mathrm{w}}\right), & \tilde{x} \in \Gamma(t), \\
-\varepsilon^{l} \tilde{D}^{\mathrm{w}} \nabla \tilde{u}^{\mathrm{w}} \cdot \nu^{\mathrm{w}}=0, & \tilde{x} \in \Gamma^{\mathrm{ws}}(t), \\
-\varepsilon^{j} \tilde{E}^{\mathrm{w}} \nabla \tilde{v}^{\mathrm{w}} \cdot \nu^{\mathrm{w}}=0, & \tilde{x} \in \Gamma^{\mathrm{w}}(t),
\end{array}
$$

Copyright $@$ by SIAM. Unauthorized reproduction of this article is prohibited. 
where $t^{\alpha} \in S^{\alpha}, \alpha \in\{\mathrm{a}, \mathrm{w}\}, t_{\mathrm{v}}^{\mathrm{w}} \in S_{\mathrm{v}}^{\mathrm{w}}$, and $t^{\mathrm{w}}=t^{\mathrm{a}} T^{\mathrm{a}} / T^{\mathrm{w}}$ and the derivatives are taken with respect to the dimensionless variables.

2.1. Choice of $\boldsymbol{k}, \boldsymbol{l}, \boldsymbol{j}$, and $\boldsymbol{m}$. System (2.7) contains four parameters introduced by the nondimensionalization: $k, l, j$, and $m$. From the nondimensionalization we get a suggestion for the choice of these parameters: In order to account for all processes optimally, it is desirable that all processes happen on the same time scale. Therefore, $k, l$, and $j$ need to be chosen such that the characteristic times of diffusion of all species are equal to that of the fastest species, which we choose as close to unity as possible, $T^{\mathrm{a}}=\max \left\{1, L^{2} / D_{\text {ref }}^{1}\right\}$. Therefore, we set $T^{\mathrm{w}}=T_{\mathrm{v}}^{\mathrm{w}}=T^{\mathrm{a}}$. This determines $k, l$, and $j$. The parameter $m$ is to be chosen such that $\tilde{a} \approx 1$. This corresponds to $C^{\mathrm{ex}} \approx \varepsilon^{m} L / T^{\mathrm{a}}$; i.e., $m$ needs to be chosen small if $C^{\mathrm{ex}}$ is large and needs to be chosen large if $C^{\mathrm{ex}}$ is small. Noting that these choices imply $S^{\mathrm{a}}=S^{\mathrm{w}}=S_{\mathrm{v}}^{\mathrm{w}}=: S$ and $\tilde{C}_{\mathrm{ex}}^{\mathrm{a}} / C^{\mathrm{H}}=\tilde{C}_{\mathrm{ex}}^{\mathrm{w}}=: \tilde{a}$, system (2.7) simplifies to

$$
\begin{array}{ll}
\partial_{t} \tilde{u}^{\mathrm{a}}-\nabla \cdot\left(\varepsilon^{k} \tilde{D}^{\mathrm{a}} \nabla \tilde{u}^{\mathrm{a}}-\tilde{w}^{\mathrm{a}} \tilde{u}^{\mathrm{a}}\right)=0, & \tilde{x} \in \Omega^{\mathrm{a}}(t), t \in S, \\
\partial_{t} \tilde{u}^{\mathrm{w}}-\nabla \cdot\left(\varepsilon^{l} \tilde{D}^{\mathrm{w}} \nabla \tilde{u}^{\mathrm{w}}-\tilde{w}^{\mathrm{w}} \tilde{u}^{\mathrm{w}}\right)=-\tilde{R}^{\mathrm{u}} \tilde{f}^{\mathrm{r}}, \quad \tilde{x} \in \Omega^{\mathrm{w}}(t), t \in S, \\
\partial_{t} \tilde{v}^{\mathrm{w}}-\nabla \cdot\left(\varepsilon^{j} \tilde{E}^{\mathrm{w}} \nabla \tilde{v}^{\mathrm{w}}-\tilde{w}^{\mathrm{w}} \tilde{v}^{\mathrm{w}}\right)=+\tilde{R}^{\mathrm{v}} \tilde{f}^{\mathrm{r}}, \quad \tilde{x} \in \Omega^{\mathrm{w}}(t), t \in S, \\
-\varepsilon^{k} \tilde{D}^{\mathrm{a}} \nabla \tilde{u}^{\mathrm{a}} \cdot \nu^{\mathrm{a}}=\varepsilon^{m} \tilde{a} C^{\mathrm{H}}\left(\tilde{u}^{\mathrm{a}}-\tilde{u}^{\mathrm{w}}\right), & \tilde{x} \in \Gamma(t), t \in S, \\
-\varepsilon^{l} \tilde{D}^{\mathrm{w}} \nabla \tilde{u}^{\mathrm{w}} \cdot \nu^{\mathrm{w}}=-\varepsilon^{m} \tilde{a}\left(\tilde{u}^{\mathrm{a}}-\tilde{u}^{\mathrm{w}}\right), & \tilde{x} \in \Gamma(t), t \in S, \\
-\varepsilon^{l} \tilde{D}^{\mathrm{w}} \nabla \tilde{u}^{\mathrm{w}} \cdot \nu^{\mathrm{w}}=0, & \tilde{x} \in \Gamma^{\mathrm{ws}}(t), t \in S, \\
-\varepsilon^{j} \tilde{E}^{\mathrm{w}} \nabla \tilde{v}^{\mathrm{w}} \cdot \nu^{\mathrm{w}}=0, & \tilde{x} \in \Gamma^{\mathrm{w}}(t), t \in S .
\end{array}
$$

Note that no assumption on the periodicity of the domains has been made so far but only the existence of macroscopic and microscopic characteristic lengths.

3. Transformation to an $\varepsilon$-periodic reference configuration. In order to apply (periodic) homogenization techniques, we employ the method of homogenization in domains with evolving microstructure [25]. It is classically not possible to account for an evolution of the microstructure using periodic homogenization, in particular if the evolution is different in (macroscopically) different places of the medium. The idea of the method employed is to consider the problem in a fixed reference geometry and use a time- and space-dependent mapping, which accounts for the evolution of the microstructure. In terms of classical continuum mechanics, this corresponds to transforming the system from being stated in the current configuration (or Eulerian description; see (2.8), e.g.) to an appropriate reference configuration (or Lagrangian description). If the reference geometry can be chosen periodic, then periodic-homogenization ideas can be applied. The method is particularly suitable for problems where the evolution of the microstructure is induced by the chemical mechanism itself [26].

At $t=0$, the heterogeneous material is assumed to be periodic with respect to a reference cell $Y=(0,1)^{n}$ scaled by a (small) scale parameter $\varepsilon$. This assumption can be relaxed - it suffices if there exists a periodic reference configuration onto which the initial geometry can be mapped by a diffeomorphism - but it simplifies the presentation considerably. The reference cell contains a solid particle, $Z^{\mathrm{s}}$, some pore water, $Z^{\mathrm{w}}$, and void (pore) space $Z^{\mathrm{a}}$ (each being an open bounded domain with Lipschitzcontinuous boundary); i.e., at $t=0$ the domain $\Omega$ is the union of a finite amount of translated versions of $\varepsilon Y$. Then, $\Omega_{\varepsilon}^{\alpha}(0)=\Omega \cap \operatorname{int} \bigcup_{k} \varepsilon \bar{Z}_{k}^{\alpha}, \alpha \in\{\mathrm{a}, \mathrm{w}, \mathrm{s}\}$, where the subscript $k$ denotes translation of the set by $k \in \mathbb{Z}^{n}$ and $\varepsilon$ indicates the $\varepsilon$-periodic 


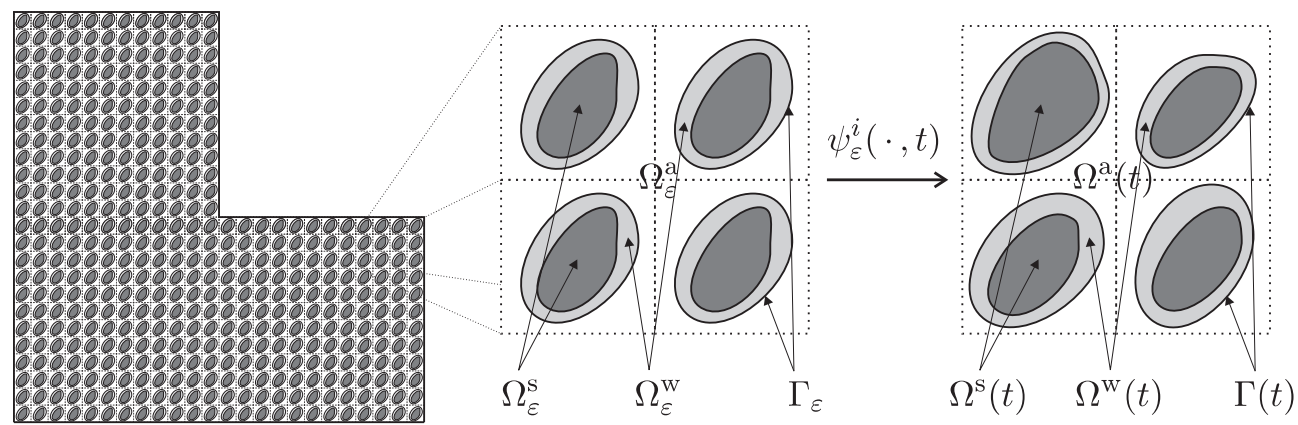

FIG. 1. Schematic cross-section of the porous medium at $t=0$ (left) with enlarged cross-section of the initial microstructure (middle). At each time $t>0$, the current spatial extent of each part of $\Omega$ is given by the image of the corresponding mapping $\psi_{\varepsilon}^{i}(\cdot, t): \Omega_{\varepsilon}^{i} \rightarrow \Omega^{i}(t), i \in\{\mathrm{a}, \mathrm{w}, \mathrm{s}\}$, so that the microstructure evolves from its initial periodic configuration (middle) to a possibly nonperiodic one for $t>0$ (right).

geometry of the domain. The evolution of the parts of $\Omega$ is assumed to be such that it can be described by orientation-preserving mappings $\psi_{\varepsilon}^{i}(\cdot, t): \Omega_{\varepsilon}^{i} \rightarrow \Omega^{i}(t)$, $i \in\{\mathrm{a}, \mathrm{w}, \mathrm{s}\}$, for each $t \in S$, whose images are the current spatial extents of pore air, pore water, and solid matrix, respectively. A schematic sketch of the setting is depicted in Figure 1. In cases where the evolution of the domain is a deformation, the corresponding function $\psi_{\varepsilon}^{i}(x, \cdot)$ gives the trajectory of the particle residing at $x$ at time $t=0$. The idea is not restricted to deformations, however, and can be used to model addition or removal of substance (increase of volume of solid matrix by precipitation, e.g.); cf. [25].

We define $\Psi_{\varepsilon}^{i}=\nabla \psi_{\varepsilon}^{i}$ and $J_{\varepsilon}^{i}=\operatorname{det} \Psi_{\varepsilon}^{i}$, which we will require to be bounded above and away from zero. In particular, these assumptions restrict the evolutions to ones where no changes of the topology of the subdomains occur and where all subdomains always have positive volume. The advantage of this construction is that the parts of $\Omega$ may evolve differently in different places. The problem in the reference configuration is posed on an $\varepsilon$-periodic medium, however, and is hence suitable for an analysis in the context of periodic homogenization.

It can be noted that $Z^{\mathrm{w}}$ and $Z^{\mathrm{s}}$ may or may not be completely contained in $Y$ so that $\Omega_{\varepsilon}^{\mathrm{w}}$ and $\Omega_{\varepsilon}^{\mathrm{s}}$ may each either be connected or not. However, $\Omega_{\varepsilon}^{\mathrm{a}}$ is assumed connected, and, for $n=2$, this implies that $\Omega_{\varepsilon}^{\mathrm{w}}$ and $\Omega_{\varepsilon}^{\mathrm{s}}$ may not be connected. If $l$ or $j$ is zero, we require $\Omega_{\varepsilon}^{\mathrm{w}}$ to be connected.

The idea of periodic homogenization (cf. $[4,33]$, e.g.) is then to examine the limit as $\varepsilon$ approaches zero in order to obtain averaged problems defined in all of $\Omega$ which are easier to treat numerically and give useful information about macroscopically observable processes.

The unknowns in the $\varepsilon$-periodic reference configuration are denoted by $u_{\varepsilon}^{\mathrm{a}}, u_{\varepsilon}^{\mathrm{w}}$, and $v_{\varepsilon}^{\mathrm{w}}$. In accordance with (2.8), we consider the model equations (plus initial conditions and homogeneous Neumann conditions at the exterior boundary for all concentrations) in the reference configuration. This can be obtained straightforwardly using some of the calculus of mechanics of deformable bodies and is given by (cf. [25])

$$
\begin{aligned}
\partial_{t}\left(J_{\varepsilon}^{\mathrm{a}} u_{\varepsilon}^{\mathrm{a}}\right)-\nabla \cdot\left(\varepsilon^{k} J_{\varepsilon}^{\mathrm{a}} \Psi_{\varepsilon}^{\mathrm{a}-1} D_{\varepsilon}^{\mathrm{a}} \Psi_{\varepsilon}^{\mathrm{a}-T} \nabla u_{\varepsilon}^{\mathrm{a}}\right) & =0, & & x \in \Omega_{\varepsilon}^{\mathrm{a}}, t \in S, \\
\partial_{t}\left(J_{\varepsilon}^{\mathrm{w}} u_{\varepsilon}^{\mathrm{w}}\right)-\nabla \cdot\left(\varepsilon^{\mathrm{l}} J_{\varepsilon}^{\mathrm{w}} \Psi_{\varepsilon}^{\mathrm{w}-1} D_{\varepsilon}^{\mathrm{w}} \Psi_{\varepsilon}^{\mathrm{w}-T} \nabla u_{\varepsilon}^{\mathrm{w}}\right) & =-r_{\varepsilon}^{\mathrm{u}} f_{\varepsilon}^{\mathrm{r}}, & & x \in \Omega_{\varepsilon}^{\mathrm{w}}, t \in S, \\
\partial_{t}\left(J_{\varepsilon}^{\mathrm{w}} v_{\varepsilon}^{\mathrm{w}}\right)-\nabla \cdot\left(\varepsilon^{j} J_{\varepsilon}^{\mathrm{w}} \Psi_{\varepsilon}^{\mathrm{w}-1} E_{\varepsilon}^{\mathrm{w}} \Psi_{\varepsilon}^{\mathrm{w}-T} \nabla v_{\varepsilon}^{\mathrm{w}}\right) & =+r_{\varepsilon}^{\mathrm{v}} f_{\varepsilon}^{\mathrm{r}}, & & x \in \Omega_{\varepsilon}^{\mathrm{w}}, t \in S,
\end{aligned}
$$


(3.1b)

$$
\begin{array}{rlrl}
-\left(\varepsilon^{k} J_{\varepsilon}^{\mathrm{a}} \Psi_{\varepsilon}^{\mathrm{a}-1} D_{\varepsilon}^{\mathrm{a}} \Psi_{\varepsilon}^{\mathrm{a}-T} \nabla u_{\varepsilon}^{\mathrm{a}}\right) \cdot \nu_{\varepsilon}^{\mathrm{a}} & =\varepsilon a_{\varepsilon} C^{\mathrm{H}}\left\|\Psi_{\varepsilon}^{\mathrm{a}-T} \nu_{\varepsilon}^{\mathrm{a}}\right\| J_{\varepsilon}^{\mathrm{a}}\left(u_{\varepsilon}^{\mathrm{a}}-u_{\varepsilon}^{\mathrm{w}}\right), & & x \in \Gamma_{\varepsilon}, t \in S, \\
-\left(\varepsilon^{l} J_{\varepsilon}^{\mathrm{w}} \Psi_{\varepsilon}^{\mathrm{w}-1} D_{\varepsilon}^{\mathrm{w}} \Psi_{\varepsilon}^{\mathrm{w}-T} \nabla u_{\varepsilon}^{\mathrm{w}}\right) \cdot \nu_{\varepsilon}^{\mathrm{w}}=-\varepsilon a_{\varepsilon}\left\|\Psi_{\varepsilon}^{\mathrm{w}-T} \nu_{\varepsilon}^{\mathrm{w}}\right\| J_{\varepsilon}^{\mathrm{w}}\left(u_{\varepsilon}^{\mathrm{a}}-u_{\varepsilon}^{\mathrm{w}}\right), & & x \in \Gamma_{\varepsilon}, t \in S, \\
-\left(\varepsilon^{l} J_{\varepsilon}^{\mathrm{w}} \Psi_{\varepsilon}^{\mathrm{w}-1} D_{\varepsilon}^{\mathrm{w}} \Psi_{\varepsilon}^{\mathrm{w}-T} \nabla u_{\varepsilon}^{\mathrm{w}}\right) \cdot \nu_{\varepsilon}^{\mathrm{w}}=0, & & x \in \Gamma_{\varepsilon}^{\mathrm{ws}}, t \in S, \\
-\left(\varepsilon^{j} J_{\varepsilon}^{\mathrm{w}} \Psi_{\varepsilon}^{\mathrm{w}-1} E_{\varepsilon}^{\mathrm{w}} \Psi_{\varepsilon}^{\mathrm{w}-T} \nabla v_{\varepsilon}^{\mathrm{w}}\right) \cdot \nu_{\varepsilon}^{\mathrm{w}}=0, & & x \in \Gamma_{\varepsilon}^{\mathrm{w}}, t \in S,
\end{array}
$$

where quantities with a subscript $\varepsilon$ in the reference configuration correspond to the same quantities with the tilde in the current configuration, $\tilde{x}=\psi_{\varepsilon}^{\mathrm{a}}(x, t), D_{\varepsilon}^{\mathrm{a}}(x, t)=$ $\tilde{D}^{\mathrm{a}}\left(\psi_{\varepsilon}^{\mathrm{a}}(x, t), t\right)$, e.g.

System (3.1) in the reference configuration appears more complicated than system (2.8) in the current configuration. The systems have the same structure, however, and all boundary conditions fit the differential equations (i.e., the fluxes in the lefthand sides of (3.1b) are exactly the fluxes in the divergence terms in (3.1a)). Most importantly, system (3.1) is stated on a fixed periodic domain and is hence suitable for a (periodic) homogenization analysis. While the system may really have become more complicated (potentially constant parameters in the current configuration, diffusivities or interfacial-exchange constants, e.g., are replaced by time- and space-dependent coefficients), the conditions on the mappings $\psi_{\varepsilon}^{i}$ ensure that these complications are of manageable technical nature in the homogenization process. An example for what the functions $\psi_{\varepsilon}^{i}$ might look like for a certain evolution can be found in section 5.2. A very important observation is that the functions $\psi_{\varepsilon}^{i}$ actually do not appear in (3.1) but only $\Psi_{\varepsilon}^{i}$ and $J_{\varepsilon}^{i}$. We will make use of this fact when coupling the evolution to the reaction-diffusion process modelling concrete carbonation in section 6 .

4. The homogenized macroproblems. The limit functions of $u_{\varepsilon}^{\mathrm{a}}, u_{\varepsilon}^{\mathrm{w}}$, and $v_{\varepsilon}^{\mathrm{w}}$ as $\varepsilon \rightarrow 0$ are denoted by $u^{\mathrm{a}}, u^{\mathrm{w}}$, and $v^{\mathrm{w}}$, respectively. The data of the problem are assumed to be given as having a slow and a fast component so that $D_{\varepsilon}^{\mathrm{a}}(x, t)=$ $D^{\mathrm{a}}(x, x / \varepsilon, t) \rightarrow D^{\mathrm{a}}(x, y, t)$ and, analogously, for $D_{\varepsilon}^{\mathrm{w}}, E_{\varepsilon}^{\mathrm{w}}, r_{\varepsilon}^{\mathrm{u}}, r_{\varepsilon}^{\mathrm{v}}, \psi_{\varepsilon}^{\mathrm{a}}, \psi_{\varepsilon}^{\mathrm{w}}$, and $a_{\varepsilon}$.

The homogenized macroproblems of (3.1) are stated below. Formally, they can be obtained straightforwardly using asymptotic expansions in powers of $\varepsilon$. Unfortunately, this ad hoc approach does not reveal under which conditions the convergence really holds. Rigorous results for problems similar to (3.1) exist, which give the assumptions to be imposed on the data as part of the process. Some discussion on this is given in section 4.2 .

4.1. The general limit problem. In order to keep the presentation concise, a generic cell problem is introduced first. The complete limit problem consists of three differential equations and boundary conditions. We state the problem for $u^{\mathrm{a}}$ first and then formulate a generic problem which applies to $u^{\mathrm{w}}$ and $v^{\mathrm{w}}$ each. Recall that $k \leq l$ since $u^{\mathrm{a}}$ is the fastest diffusing species. To keep the presentation concise, we restrict ourselves to $m \geq 0$ and $k<2$ (the omitted extreme cases can be found using the results from [29]). Note that $k=2$ would imply that all processes actually happen on the pore scale.

For this purpose, we first define a generic cell problem: Let $\varsigma_{j}, j=1, \ldots, N$, be the $Y$-periodic solution of the cell problem

$$
\begin{aligned}
& -\nabla_{y} \cdot\left(J^{\alpha} \Psi^{\alpha-1} A(x, y, t) \Psi^{\alpha-T}\left(\nabla_{y} \varsigma_{j}(x, y, t)+e_{j}\right)\right)=0, \quad y \in Z^{\alpha}, \\
& -J^{\alpha} \Psi^{\alpha-1} A(x, y, t) \Psi^{\alpha-T}\left(\nabla_{y}\left(\varsigma_{j}(x, y, t)+e_{j}\right)\right) \cdot \nu^{\alpha}=0, \quad y \in \partial Z^{\alpha} \backslash \partial Y,
\end{aligned}
$$

$x \in \Omega, t \in S$. This allows the definition of the tensors $P^{\alpha}=\left[p_{i j}^{\alpha}\right]_{i j}$ via 


$$
p_{i j}^{\alpha}(x, t)=\int_{Z^{\alpha}} J^{\alpha} \Psi^{\alpha-1} A(x, y, t) \Psi^{\alpha-T}\left(\delta_{i j}+\partial_{y_{i}} \varsigma_{j}(x, y, t)\right) \mathrm{d} y .
$$

This tensor is the macroscopic diffusion tensor, which describes the macroscopically observed diffusion properties. It encodes the microscopic diffusion properties as well as the microscopic structure and its evolution. We will specify $A$ and $\alpha$ as is required below.

Setting $\theta(0)=1$ and 0 elsewhere, the problem for $u^{\mathrm{a}}$ is given by

$\partial_{t}\left(\int_{Z^{\mathrm{a}}} J^{\mathrm{a}} \mathrm{d} y u^{\mathrm{a}}(x, t)\right)-\theta(k) \nabla \cdot\left(P^{\mathrm{a}} \nabla u^{\mathrm{a}}\right)=-\int_{\Gamma} J^{\mathrm{a}}\left\|\Psi^{\mathrm{a}-T} \nu^{\mathrm{a}}\right\| f^{\mathrm{ex}} \mathrm{d} y, \quad x \in \Omega, t \in S$,

where $f^{\text {ex }}$ is given by $f^{\text {ex }}=a\left(u^{\mathrm{a}}-u^{\mathrm{w}}\right)$ if $m=1$ and $f^{\mathrm{ex}}=D^{\mathrm{w}} \Psi^{\mathrm{w}-T} \nabla_{y} u^{\mathrm{w}} \cdot \nu^{\mathrm{w}}$ if $m<1$ and $P^{\mathrm{a}}$ is defined by (4.1), (4.2) with $A=D^{\mathrm{a}}$ and $\alpha=\mathrm{a}$. A homogeneous Neumann condition applies at the exterior boundary, and the initial condition is $u^{\mathrm{a}}(0)=u_{0}^{\mathrm{a}}$.

For $u^{\mathrm{w}}$ and $v^{\mathrm{w}}$, we define a generic limit problem for a function $w$. The complete problem for $u^{\mathrm{w}}$ and $v^{\mathrm{w}}$ consists of the problem for $w=u^{\mathrm{w}}$ and $w=v^{\mathrm{w}}$. Let $\lambda=l$ if $w=u^{\mathrm{w}}$ and $\lambda=j$ if $w=v^{\mathrm{w}}$. Moreover, $f^{\mathrm{ex}}=a\left(u^{\mathrm{a}}-u^{\mathrm{w}}\right)$ if $w=u^{\mathrm{w}}$ and $m=1$ and zero in all other cases. The function $r$ is given by $r^{\mathrm{u}}$ for $w=u$ and $r^{\mathrm{v}}$ for $w=v$. If $\lambda<2$, the limit problem is given by

$$
\begin{aligned}
& \partial_{t}\left(\int_{Z^{\mathrm{w}}} J^{\mathrm{w}} \mathrm{d} y w\right.(x, t))-\theta(\lambda) \nabla \cdot\left(P^{\mathrm{w}} \nabla w\right) \\
&=\int_{Z^{\mathrm{w}}} J^{\mathrm{w}} r f^{\mathrm{r}} \mathrm{d} y+\int_{\Gamma} J^{\mathrm{w}}\left\|\Psi^{\mathrm{w}-T} \nu^{\mathrm{w}}\right\| f^{\mathrm{ex}} \mathrm{d} y, \quad x \in \Omega, t \in S,
\end{aligned}
$$

together with a homogeneous Neumann condition at the exterior boundary and initial condition $w(0)=w_{0}$. The effective diffusion tensor $P^{\mathrm{w}}$ is defined by (4.1), (4.2) with $\alpha=\mathrm{w}$ and $A=D^{\mathrm{w}}$ if $w=u^{\mathrm{w}}$ and $A=E^{\mathrm{w}}$ if $w=v^{\mathrm{w}}$. If $\lambda=2$, the limit equation is given by

$$
\partial_{t}\left(J^{\mathrm{w}} w(x, y, t)\right)-\nabla_{y} \cdot\left(J^{\mathrm{w}} \Psi^{\mathrm{w}-1} A \Psi^{\mathrm{w}-T} \nabla_{y} w\right)=J^{\mathrm{w}} r f^{\mathrm{r}}, \quad y \in Z^{\mathrm{w}}, x \in \Omega, t \in S .
$$

For $w=u^{\mathrm{w}}$, the boundary condition on $\Gamma$ is

$$
\begin{aligned}
-J^{\mathrm{w}} \Psi^{\mathrm{w}-1} D^{\mathrm{w}} \Psi^{\mathrm{w}-T} \nabla_{y} u^{\mathrm{w}} \cdot \nu^{\mathrm{w}} & =-a\left(u^{\mathrm{a}}-u^{\mathrm{w}}\right) & & \text { if } m=1, \\
u^{\mathrm{w}} & =u^{\mathrm{a}} & & \text { if } m<1 .
\end{aligned}
$$

Homogeneous Neumann conditions apply for $w=u^{\mathrm{w}}$ on $\Gamma^{\mathrm{ws}}$ and for $w=v^{\mathrm{w}}$ on $\Gamma^{\mathrm{w}}$. Note that for $l<2$ and $m<1$, boundary condition (4.7) implies that it makes sense to define a combined concentration $u=\left|Z^{\mathrm{a}}\right| u^{\mathrm{a}}+\left|Z^{\mathrm{w}}\right| u^{\mathrm{w}}$, which satisfies an equation obtained by adding the limit equations for $u^{\mathrm{a}}$ and $u^{\mathrm{w}}$.

4.2. Some remarks on rigorous results. The limit problems can be obtained formally using asymptotic expansions, e.g. For the rigorous proof of convergence of the sequences of solutions of (3.1) to the solutions of the above limit problems, periodic-homogenization techniques $[4,33]$ can be used. Some difficulties arise from the nonlinear concentration-dependent reaction term. For concentration-independent reaction terms, the convergence is proven for the problem with $J^{\alpha} \equiv 1$ (i.e., no volume changes) in [29] and for a similar problem with evolving volume factors in [27]; also see [26]. Combining the techniques used there, existence of solutions of the $\varepsilon$-periodic 
microproblems and the convergence to the solutions of the above limit problems follow for concentration-independent reaction terms without any additional ideas.

A similar problem to (3.1) with nonlinear concentration-dependent reaction rates is considered in [24]. Compared to the concentration-independent reaction-rate case, difficulties arise in the proof of existence of solutions of the $\varepsilon$-problems and in obtaining sufficiently strong a priori estimates allowing the limit passage in the nonlinear reaction terms. Using the methods described in [24], existence of solutions of the $\varepsilon$-problems can be shown if $r^{\mathrm{v}}$ is a linearly bounded function. In order to obtain sufficiently strong a priori estimates allowing the limit passage, several further sufficient conditions can be given. For example, $l=0$ or $r^{\mathrm{u}}$ being independent of $u_{\varepsilon}^{\mathrm{w}}$ in the equation for $v_{\varepsilon}^{\mathrm{w}}$ and being a linear function in the equation for $u_{\varepsilon}^{\mathrm{w}}$ suffices. In all cases considered in [24], the limit of the concentration-dependent part of the reaction term in the equations for $u^{\mathrm{w}}$ and $v^{\mathrm{w}}$ is given by $f^{\mathrm{r}}=r_{\mathrm{u}}\left(u^{\mathrm{w}}\right) r_{\mathrm{v}}\left(v^{\mathrm{w}}\right)$. In what follows, we assume that this is the limit in all cases considered here.

Some further remarks on the prerequisites on the other data required in the convergence proofs are appropriate. The coefficient functions $J^{\alpha} \Psi^{\alpha-1} D^{\alpha} \Psi^{\alpha-T}, \alpha \in$ $\{\mathrm{a}, \mathrm{w}\}$, need to be admissible test functions in the sense of two-scale convergence [2]. This is the case if these functions are continuous with respect to the slow or the fast variable, e.g.

4.3. Macroscopic diffusion tensors. It is convenient to define the effective diffusion tensors without an evolution of the microstructure and with unit diffusivity. In order to avoid confusion with the notation of the previous sections, we denote these tensors by $Q$. Then, only the solutions of two cell problems are required: Let $\varsigma_{j}^{\alpha}, j=1, \ldots, n$, be the $Y$-periodic solution of the cell problem

$$
\begin{aligned}
& -\nabla_{y} \cdot\left(\nabla_{y} \varsigma_{j}^{\alpha}(y)+e_{j}\right)=0, \quad y \in Z^{\alpha}, \\
& -\left(\nabla_{y} \varsigma_{j}^{\alpha}(y)+e_{j}\right) \cdot \nu^{\alpha}=0, \quad y \in \partial Z^{\alpha} \backslash \partial Y,
\end{aligned}
$$

the weak form of which is given by

$$
\left(\nabla \varsigma_{j}^{\alpha}+e_{j} \mid \nabla \phi\right)_{Z^{\alpha}}=0
$$

for all $Y$-periodic test functions $\phi, \alpha \in\{\mathrm{a}, \mathrm{w}\}$. The macroscopic diffusion tensors are then given by $Q^{\alpha}=\left[q_{j k}^{\alpha}\right]_{j k}$ via

$$
q_{j k}^{\alpha}=\frac{1}{\left|Z^{\alpha}\right|} \int_{Z^{\alpha}}\left(\delta_{j k}+\partial_{y_{j}} \varsigma_{k}^{\alpha}(y)\right) \mathrm{d} y,
$$

where $\delta_{j k}$ is the Kronecker delta. These tensors are symmetric and positive definite (cf. [8], e.g.). Note that for $J^{\alpha} \Psi^{\alpha-1} D^{\alpha} \Psi^{\alpha-T}$ dependent only on the macrovariable, we recover the notation from the previous section via $P^{\alpha}=\left|Z^{\alpha}\right| J^{\alpha} \Psi^{\alpha-1} D^{\alpha} \Psi^{\alpha-T} Q^{\alpha}$ and $P_{\mathrm{v}}^{\mathrm{w}}=\left|Z^{\mathrm{w}}\right| \Psi^{\mathrm{w}-1} E^{\mathrm{w}} \Psi^{\mathrm{w}-T} Q^{\mathrm{w}}$.

4.4. Numerical implementation. We briefly discuss how the system of partial differential equations is solved numerically. We use the finite element method and assume the macroscopic sample to extend uniformly in one direction far enough for a two-dimensional approximation to be valid; i.e., we consider two-dimensional crosssections.

Two types of reference cells are considered in the next section. In the simulations illustrating the influence of scaling (section 5.1), the microstructure is assumed to be of ball type as depicted in Figure 2. This allows the simplification to one space 


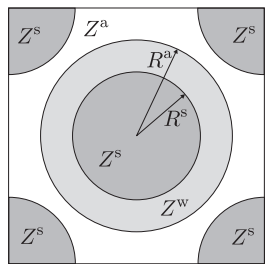

FIG. 2. Schematic cross-sections of the cells used in the simulations.

dimension using spherical coordinates.

For the illustration of the influence of the evolving microstructure (section 5.2), a two-dimensional cuboid microstructure is used in order to show this for a more general case; also cf. Figures 10 and 11.

For the discretization of the macroscopic variable, we triangulate the domain $\Omega$ and use linear splines as test and trial functions. The resulting system of ordinary differential equations in time is solved using the MATLAB solver ode15s, an implicit variable-order solver based on numerical differentiation formulas.

If $l$ or $j$ is equal to two, we also need to solve partial differential equations in the reference cell, which are coupled to the equations with respect to the macrovariable. For the problems with the ball-type microstructure depicted in Figure 2, the problem is first converted to spherical coordinates. In any case, we also use linear test and trial functions for these equations and solve the resulting system of ordinary differential equations in time using the MATLAB solver ode15s. This is done for each macroscopic time step.

5. Numerical comparison of some limit models. In order to show the appropriateness of the nondimensionalization as well as of the obtained limit problems, we conduct some numerical experiments. We begin with investigating the influence of the scaling exponents neglecting the influence of the evolution of the microstructure, i.e., $\psi_{\varepsilon}^{i}=\mathrm{Id}, i \in\{\mathrm{a}, \mathrm{w}, \mathrm{s}\}$, and then consider results for a prototypical model accounting for an evolving microstructure.

5.1. Influence of scaling. It was theoretically argued in section 2 that the characteristic lengths and times of the problem should be accounted for in the homogenization process by scaling the material parameters with powers of the homogenization parameter $\varepsilon$. The appropriate scaling powers were found from a nondimensionalization. In what follows, we conduct some numerical experiments for simple test problems. It is shown that the limit problems corresponding to correct scalings resolve the important processes well while bad choices of scaling lead to poor limit models.

Diffusion. We discuss the influence of the choice of scaling exponents on diffusion for a very much simplified problem for only one substance which is only diffusing; i.e., we consider the equation for $u_{\varepsilon}^{\mathrm{w}}$ with $a=0$. Assume $\Omega$ to be a square with side length 1, i.e., $\Omega=(0,1)^{2}, S=(0,1)$, and $u_{\varepsilon}^{\mathrm{w}}(x, 0)=\mathrm{e}^{-10\left\|x-(1 / 2,1 / 2)^{T}\right\|^{2}}$. Clearly, we choose $u_{\mathrm{ref}}^{\mathrm{w}}=1$ and write $u=u^{\mathrm{w}}$ for simplicity.

We take $L=1$ and assume the medium has a ball-type microstructure as depicted in Figure 2 such that $\ell=10^{-4}$ and $\ell_{2}^{\mathrm{D}}=\ell / \sqrt{10}$ are reasonable choices for the characteristic microscopic lengths. Thus, the tensor $Q^{\mathrm{w}}$ is diagonal with all diagonal elements equal. Since the exact value is not important here, we assume $Q^{\mathrm{w}}=0.05$. We consider the two cases 

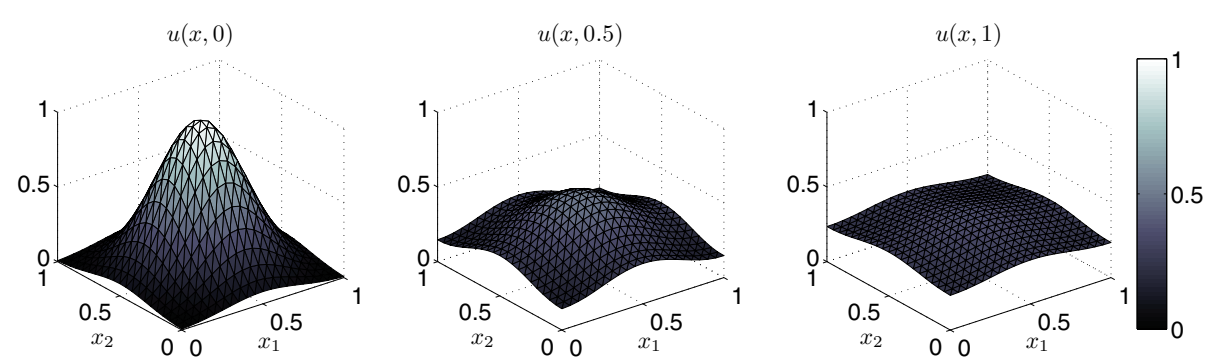

FIG. 3. Solution to problem (5.1) for $t=0$ (left), $t=0.5$ (middle), and $t=1$ (right).

1. $D^{2}=1$,

2. $D^{2}=10^{-9}$.

It is clear that we expect the important mechanism to be macroscopic diffusion in case 1 and microscopic diffusion in case 2. According to the nondimensionalization (cf. section 2), we need to choose

1a. $l=0$ for case 1 ,

2a. $l=2$ for case 2 ,

since then $T^{\mathrm{w}}=1$ in both cases. To show the appropriateness of these choices we also consider the opposite cases, i.e.,

1b. $l=2$ (implying $T^{\mathrm{w}}=10^{-9}$ ) for case 1 ,

2b. $l=0$ (implying $T^{\mathrm{w}}=10^{9}$ ) for case 2 .

Since these choices are bad in terms of what the nondimensionalization suggests, we expect the corresponding limit models to resolve the important mechanism poorly.

Reading off the limit problem of section 4.1 , in case 1a, we have to solve

$$
\begin{aligned}
\partial_{t} u(x, t)-0.05 \Delta u & =0, & & x \in \Omega, t \in(0,1), \\
-0.05 \nabla u \cdot \nu & =0, & & x \in \partial \Omega, t \in(0,1), \\
u(x, 0) & =\mathrm{e}^{-10\left\|x-(1 / 2,1 / 2)^{T}\right\|^{2}}, & & x \in(0,1)^{2} .
\end{aligned}
$$

The solution to this problem is shown in Figure 3. It can be seen that the macroscopic diffusion is captured well.

On the other hand, in case 1b, the limit model captures only diffusion in the cell, i.e., in $Y$. Since the initial value is a function of $x$ only and does not depend on the microvariable, i.e., on $x / \varepsilon$, and there are no mechanisms disrupting this initial state, we do not have diffusion and, hence, $u(x, t)=u(x, 0)$. Clearly, this limit model is inappropriate. Even if we had chosen an initial value which is not constant in each cell, no macroscopic diffusion would have taken place since the limit model does not include this mechanism.

Similar to case $1 \mathrm{~b}$, the limit model for case 2 a gives $u(x, t)=u(x, 0)$, which is a reasonable model since no observable diffusion is expected in this case. On the other hand, for case $2 \mathrm{~b}$, we have to solve

$$
\begin{aligned}
\partial_{t} u(x, t)-0.05 \Delta u & =0, & & x \in \Omega, t \in\left(0,10^{-9}\right), \\
-0.05 \nabla u \cdot \nu & =0, & & x \in \partial \Omega, t \in\left(0,10^{-9}\right), \\
u(x, 0) & =\mathrm{e}^{-10\left\|x-(1 / 2,1 / 2)^{T}\right\|^{2},} & & x \in(0,1)^{2} .
\end{aligned}
$$

Owing to the different time scales, $t=10^{-9}$ in problem (5.2) corresponds to the same (dimensional) instant in time as $t=1$ in problem (5.1). In Figure 4, the 

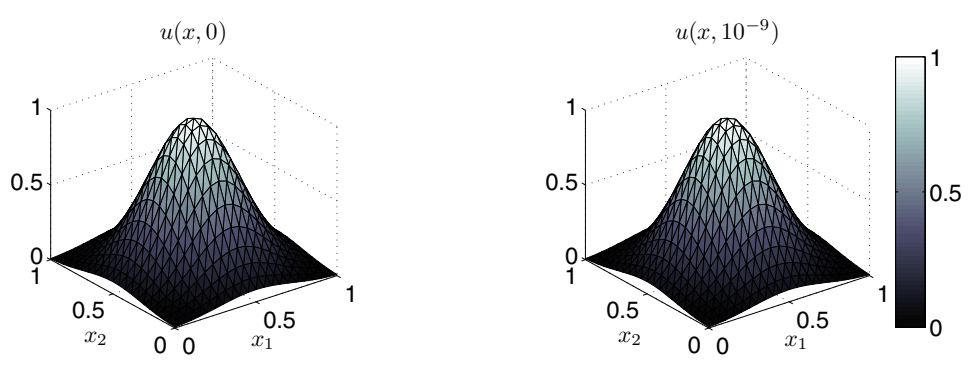

FiG. 4. Solution to problem (5.2) for $t=0$ (left) and $t=10^{-9}$ (right).

solution to problem (5.2) is plotted. It can be seen that there is virtually no change of concentration in the time interval of consideration. This agrees with what was obtained for case $2 \mathrm{a}$, but it was immediately clear there.

Things are even more extreme if the initial value depends on the microscopic scale, i.e., $\bar{u}_{0}=\bar{u}_{0}(x, y)$. Then, in case 1a, we have to solve the same problem since the initial value is then given by $u_{0}=\int_{Y} \bar{u}_{0}(x, y) \mathrm{d} y$. This is a reasonable model since diffusion on the microscopic scale is so fast in this case that the local diffusion equilibrium in the cell is reached almost instantaneously (consider a diffusivity of $1 \mathrm{~cm}^{2} / \mathrm{d}$ on a length of $10^{-4.5} \mathrm{~cm}$ and a time interval of $(0,1) \mathrm{d}$, where $\mathrm{d}$ stands for day). Model $1 \mathrm{~b}$ would have resolved the diffusion in the cell; it does not resolve the macroscopic diffusion, however, and is therefore inappropriate.

In case 2, microscopic diffusion is important. Model 2a resolves this diffusion and, hence, is the appropriate model. Model $2 \mathrm{~b}$ would have given the same output as before which does not resolve the diffusion on the microscopic scale. It does resolve the macroscopic output correctly in this case. However, if other mechanisms are also important, interfacial exchange for example, this is not the case, as can be seen in the next example.

Interfacial exchange. For this example, we assume we have two diffusing concentrations, each given in a different part of the porous medium. We assume the general setting to be as in the previous example and choose the unit cell to be as depicted in Figure 2, i.e., a ball-type microstructure. In the simulations, we choose $\left(R^{\mathrm{a}}\right)^{3}=3 /(8 \pi)$ and $R^{\mathrm{s}}=0$ and assume that $\left|Z^{\mathrm{s}}\right|=0$. Note that this yields $\left|Z^{\mathrm{a}}\right|=\left|Z^{\mathrm{w}}\right|=1 / 2$.

We assume the setting for the first concentration, $u^{\mathrm{a}}$, as in case 1 of the previous example. For the second concentration, $u^{\mathrm{w}}$, we assume a diffusivity of $10^{-9} \mathrm{~cm}^{2} / \mathrm{d}$ and $\ell_{2}^{\mathrm{D}}=\ell / \sqrt{10}$ (note that this corresponds to case 2 of the previous example) and we assume $C^{\mathrm{H}}=1$ and $C^{\mathrm{ex}}=10^{-4} \mathrm{~cm} / \mathrm{d}$ for now. Therefore, the scaling exponents suggested by the nondimensionalization are $k=0, l=2$, and $m=1$. The limit problem is given by

$$
\partial_{t} u^{\mathrm{a}}(x, t)-0.05 \Delta u^{\mathrm{a}}=-\frac{1}{\left|Z^{\mathrm{a}}\right|} \int_{\Gamma}\left(u^{\mathrm{a}}-u^{\mathrm{w}}\right) \mathrm{d} y, \quad x \in \Omega, t \in(0,1),
$$

$\partial_{t} u^{\mathrm{w}}(x, y, t)-0.1 \Delta_{y} u^{\mathrm{w}}=0$

$$
\begin{aligned}
& -0.1 \nabla_{y} u^{\mathrm{w}} \cdot \nu^{\mathrm{w}}=-\left(u^{\mathrm{a}}-u^{\mathrm{w}}\right), \\
& -0.1 \nabla_{y} u^{\mathrm{w}} \cdot \nu^{\mathrm{w}}=0,
\end{aligned}
$$$$
-\nabla u^{\mathrm{a}} \cdot \nu=0,
$$ 

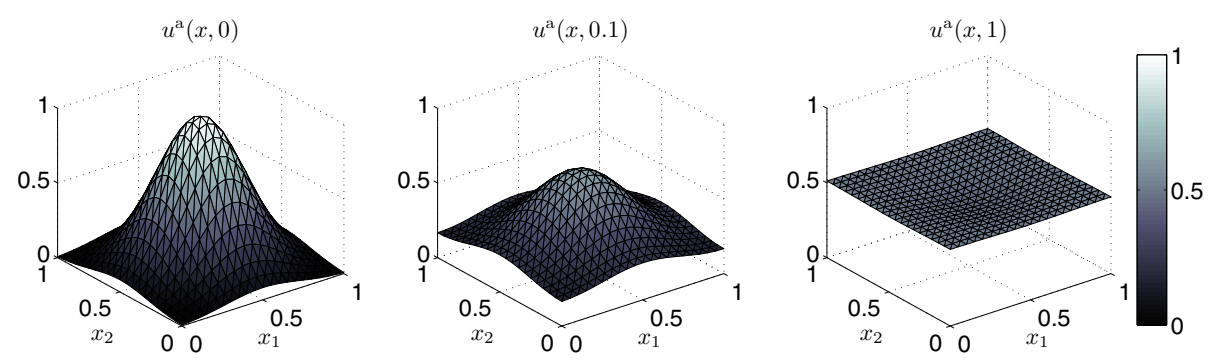

Fig. 5. Profiles of $u^{\mathrm{a}}$ in problem (5.3) for $t=0$ (left), $t=0.1$ (middle), and $t=1$ (right).
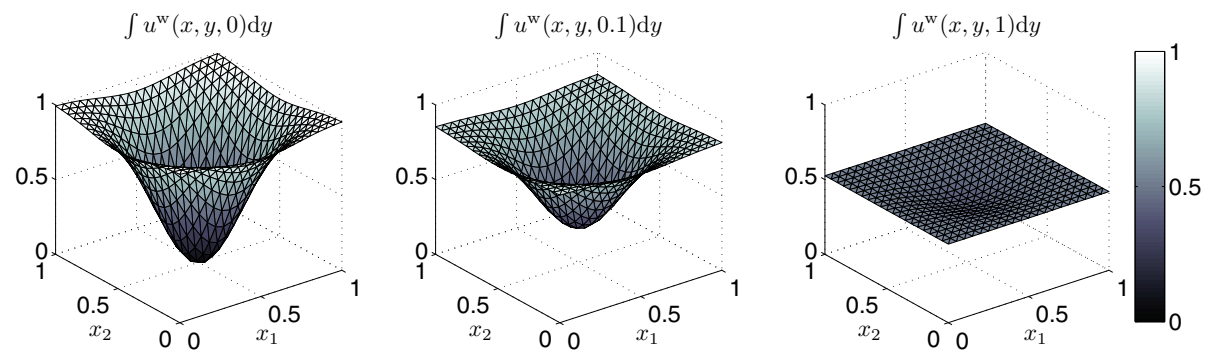

Fig. 6. Profiles of $u^{\mathrm{w}}$ in problem (5.3) for $t=0$ (left), $t=0.1$ (middle), and $t=1$ (right).
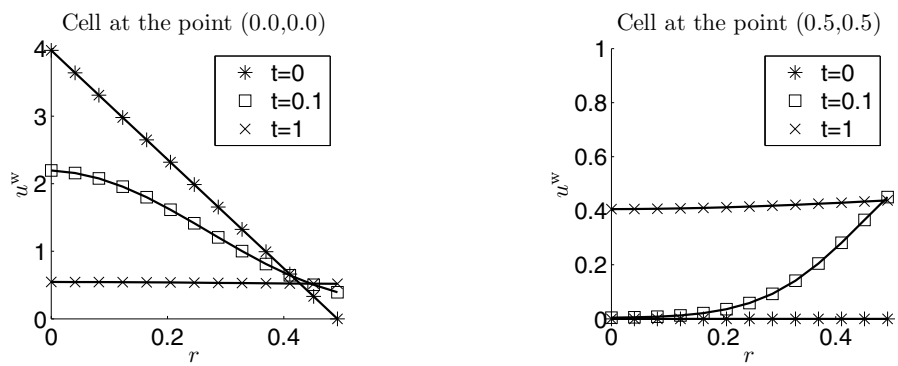

FIG. 7. Cell solutions to (5.3) in two different cells. Cell at $(0,0)$ (left) and at $(0.5,0.5)$ (right).

which is a classic distributed-microstructure model; cf. [12], e.g. For the initial conditions, we choose $u^{\mathrm{a}}(x, 0)=\mathrm{e}^{-10\left\|x-(1 / 2,1 / 2)^{T}\right\|^{2}}$ as before and $u^{\mathrm{w}}(x, y, 0)=$ $K\left(1-\mathrm{e}^{-10\left\|x-(1 / 2,1 / 2)^{T}\right\|^{2}}\right)\left(R^{\mathrm{a}}-r\right) /\left(R^{\mathrm{a}}-R^{\mathrm{s}}\right)$ with

$$
K=\left(1-\frac{4}{3} \pi\left(R^{\mathrm{a}}\right)^{3}\right)\left(\pi \frac{\left(R^{\mathrm{s}}\right)^{4}-\left(R^{\mathrm{a}}\right)^{4}}{R^{\mathrm{a}}-R^{\mathrm{s}}}+\frac{4 \pi R^{\mathrm{a}}}{3} \frac{\left(R^{\mathrm{a}}\right)^{3}-\left(R^{\mathrm{s}}\right)^{3}}{R^{\mathrm{a}}-R^{\mathrm{s}}}\right)^{-1} .
$$

Note that we have $\int_{Z^{\mathrm{w}}} u^{\mathrm{w}}(x, y, 0)=\left|Z^{\mathrm{a}}\right|\left(1-u^{\mathrm{a}}(x, 0)\right)$. The solution to (5.3) is plotted in Figures 5-7. As can be seen, all important processes, i.e., macroscopic diffusion of $u^{\mathrm{a}}$, microscopic diffusion of $u^{\mathrm{w}}$, and the exchange at the internal interface, are resolved well. In particular, it can be seen in Figure 7(left) that it takes time for $u^{\mathrm{w}}$ to diffuse from the center of $Z^{\mathrm{w}}$ (where most of $u^{\mathrm{w}}$ is located at $t=0$ ) to the boundary $\Gamma$ where the equalization takes place.

For comparison, we also plot the solution to the limit problem corresponding to the inappropriate choice $l=0$ (cf. Figures 8 and 9 ), which leads to a model of parallelflow type. From a comparison of the middle plots of Figures 5 and 8 as well as of Figures 6 and 8 , it can be seen that the important process in the cell, that is, the 

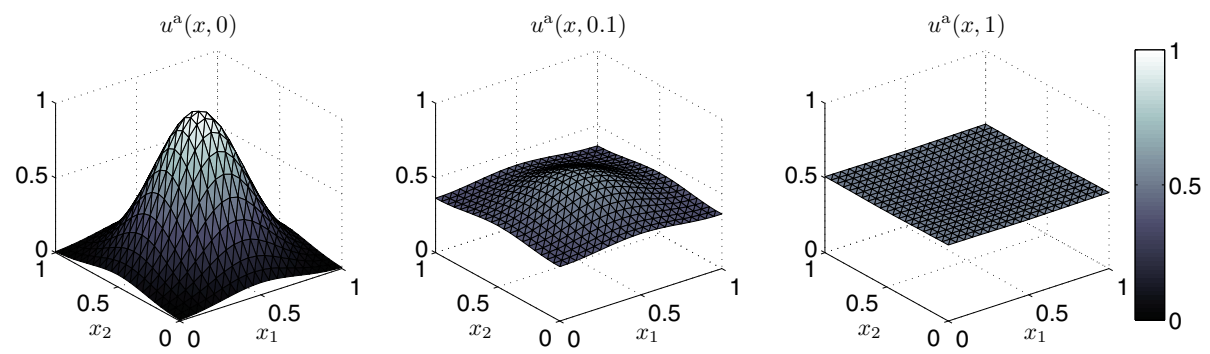

Fig. 8. Profiles of $u^{\mathrm{a}}$ for $t=0$ (left), $t=0.1$ (middle), and $t=1$ (right) for the problem corresponding to the choice $l=0$.
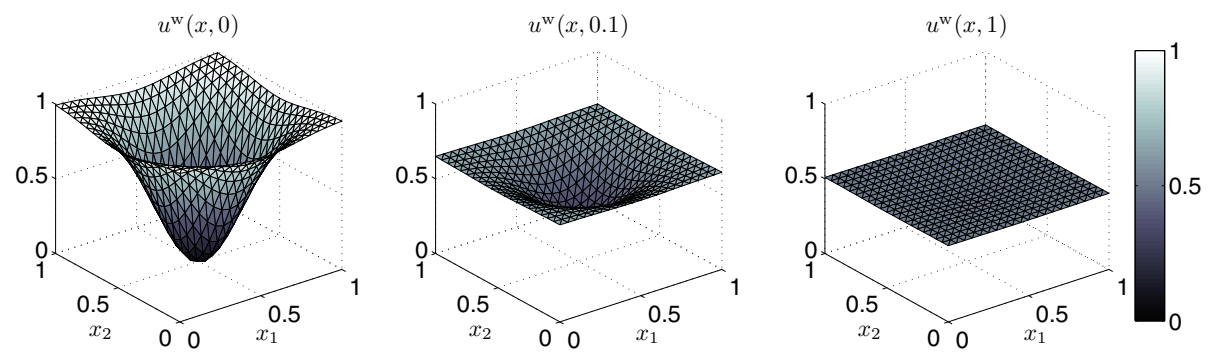

FIG. 9. Profiles of $u^{\mathrm{w}}$ for $t=0$ (left), $t=0.1$ (middle), and $t=1$ (right) for the problem corresponding to the choice $l=0$.

diffusion from the center of $Z^{\mathrm{w}}$ to $\Gamma$, is not captured accurately. The delay caused by this transport is not captured in the model with $l=0$ ! Of course, the equilibrium state, which the system tends to, is the same. In other words, the general macroscopic processes are captured, but the processes on the microscopic scale, which have a strong influence on the macroscopic processes in this example, are not resolved. Note that, since $\Omega_{\varepsilon}^{\mathrm{w}}$ is disconnected for the microstructure chosen, we do not obtain macroscopic diffusion in the limit even though $l=0$. For comparison, we still investigate the limit problem with macroscopic diffusion and disconnected microstructure.

It can be seen that if $C^{\mathrm{ex}}$ was considerably larger, $C^{\mathrm{ex}}=1 \mathrm{~cm} / \mathrm{d}$ for example, the interfacial exchange would have been so fast that the equilibrium at the interface, i.e., $u^{\mathrm{a}}=u^{\mathrm{w}}$, would have been reached almost instantaneously and remained that way (not plotted). For this value of $C^{\mathrm{ex}}$, the nondimensionalization suggests the scaling exponent to be $m=0$, which, in turn, corresponds to the Dirichlet condition $u^{\mathrm{a}}=u^{\mathrm{w}}$ at the interface in the homogenization limit. On the other hand, if $C^{\mathrm{ex}}$ is considerably smaller than $10^{-4} \mathrm{~cm} / \mathrm{d}$ in the above example, $10^{-8} \mathrm{~cm} / \mathrm{d}$ say, the interfacial exchange is almost negligible on the time scale under consideration (not plotted). In this case, the nondimensionalization suggests a scaling exponent of $m=2$, which, in the homogenization limit, corresponds to a homogeneous Neumann condition at the interface; i.e., there is no flux across the interface. Hence, in both cases, the homogenized models arising from the micromodels scaled as suggested by the nondimensionalization capture the relevant processes best.

5.2. Influence of evolving microstructure. Using a simple test problem, we want to illustrate how the evolution of the microstructure influences the model output. For this investigation, we use a two-dimensional rectangular microstructure of a shrinking square. In order to focus on the aspect of the evolution of the microstruc- 
ture, the reference cell again consists of only two phases. In the implementation, the rectangles are discretized in the same way as the macroscopic domain.

In particular, the particle in the reference cell is modelled by a cuboid centered at the cell midpoint, which shrinks over time. The parts of the unit cell $Y$ can then be modelled as

$$
\begin{aligned}
Z^{\mathrm{w}}(t) & =(a(t), 1-a(t)) \times(b(t), 1-b(t)), \\
Z^{\mathrm{a}}(t) & =(0,1)^{2} \backslash \bar{Z}^{\mathrm{w}}(t),
\end{aligned}
$$

with functions $0<a, b<1 / 2$. Setting $\Omega=(0,1)^{2}$ and defining the domains $\Omega_{\varepsilon}^{\mathrm{a}}(t)$ and $\Omega_{\varepsilon}^{\mathrm{w}}(t)$ in the usual way (note that $\Omega_{\varepsilon}^{\mathrm{s}}(t) \equiv \emptyset$ ), we obtain a time-dependent domain with evolving microstructure, which has the same evolution of the microstructure in each cell. The evolution can be made different in each cell by allowing the functions $a$ and $b$ to depend on the cell. The question is now to find transformations $\psi_{\varepsilon}^{\mathrm{a}}$ and $\psi_{\varepsilon}^{\mathrm{w}}$ which map the reference domains to the current configuration. Of course, there are a variety of reasonable options since we do not require the functions $\psi_{\varepsilon}^{i}$ to satisfy any equation or other restrictions. Here, we use affine-linear transformations.

We want to consider one possible choice for $\psi_{\varepsilon}^{\mathrm{a}}$ and $\psi_{\varepsilon}^{\mathrm{w}}$ for the situation introduced above. For this purpose, we begin with defining the mapping $\psi_{x}^{\mathrm{w}}: Z^{\mathrm{w}}(0) \rightarrow Z^{\mathrm{w}}(t)$ for one unit cell. The subscript $x$ is introduced for future use. Its function is to determine the cell for which the transformation is intended. We further denote by $[x]_{Y}$ the unique integer combination $\sum_{i=1}^{n} k_{i} e_{i}$ of the periods such that $\{x\}_{Y}=x-[x]_{Y}$ belongs to $[0,1)^{n}$ (where $e_{i}$ is the $i$ th unit vector).

Again, we use standard letters for the coordinates in the reference configuration and letters with a tilde for the coordinates in the current configuration. A possible choice for $\psi_{x}^{\mathrm{w}}$ is

$$
\begin{aligned}
& \left(x_{1}, x_{2}\right) \mapsto\left(\tilde{x}_{1}, \tilde{x}_{2}\right)=\left(\psi_{x, 1}^{\mathrm{w}}\left(x_{1}, x_{2}\right), \psi_{x, 2}^{\mathrm{w}}\left(x_{1}, x_{2}\right)\right) \\
& \quad=\left(x_{1}+\left(\frac{a_{x}(t)-1 / 2}{a_{x}(0)-1 / 2}-1\right)\left(x_{1}-1 / 2\right), x_{2}+\left(\frac{b_{x}(t)-1 / 2}{b_{x}(0)-1 / 2}-1\right)\left(x_{2}-1 / 2\right)\right) .
\end{aligned}
$$

Note that the parameter $x$ of $\psi_{x}^{\mathrm{w}}$ appears only in $a_{x}$ and $b_{x}$ and that the $j$ th component $\psi_{x, j}^{\mathrm{w}}$ is actually dependent only on $x_{j}$, i.e., $\psi_{x, j}^{\mathrm{w}}=\psi_{x, j}^{\mathrm{w}}\left(x_{j}\right)$. Moreover, the scale splitting as required in section 4 is directly given here: The parameter $x$ and $\varepsilon[x / \varepsilon]_{Y}$ belong to the slow component while $\{x / \varepsilon\}_{Y}$ is the fast component. The transformation for the unit cell can be easily modified to obtain the transformation $\psi_{\varepsilon}^{\mathrm{w}}: \Omega_{\varepsilon}^{\mathrm{w}}(0) \rightarrow \Omega_{\varepsilon}^{\mathrm{w}}(t)$ for the $\varepsilon$-periodic domain,

$$
\begin{aligned}
\left(\tilde{x}_{1}, \tilde{x}_{2}\right)= & \left(\varepsilon\left[x_{1} / \varepsilon\right]_{Y}, \varepsilon\left[x_{2} / \varepsilon\right]_{Y}\right) \\
& +\varepsilon\left(\left\{x_{1} / \varepsilon\right\}_{Y}+\left(\frac{a_{x}(t)-1 / 2}{a_{x}(0)-1 / 2}-1\right)\left(\left\{x_{1} / \varepsilon\right\}_{Y}-1 / 2\right),\right. \\
& \left.\left\{x_{2} / \varepsilon\right\}_{Y}+\left(\frac{b_{x}(t)-1 / 2}{b_{x}(0)-1 / 2}-1\right)\left(\left\{x_{2} / \varepsilon\right\}_{Y}-1 / 2\right)\right),
\end{aligned}
$$

or, in a simpler way,

$$
\psi_{\varepsilon}^{\mathrm{w}}\left(x_{1}, x_{2}\right)=\varepsilon\left(\left[x_{1} / \varepsilon\right]_{Y}+\psi_{x, 1}^{\mathrm{w}}\left(\left\{x_{1} / \varepsilon\right\}_{Y}\right),\left[x_{2} / \varepsilon\right]_{Y}+\psi_{x, 2}^{\mathrm{s}}\left(\left\{x_{2} / \varepsilon\right\}_{Y}\right)\right) .
$$

The functioning of the mapping is illustrated in Figure 10 for the choice of 

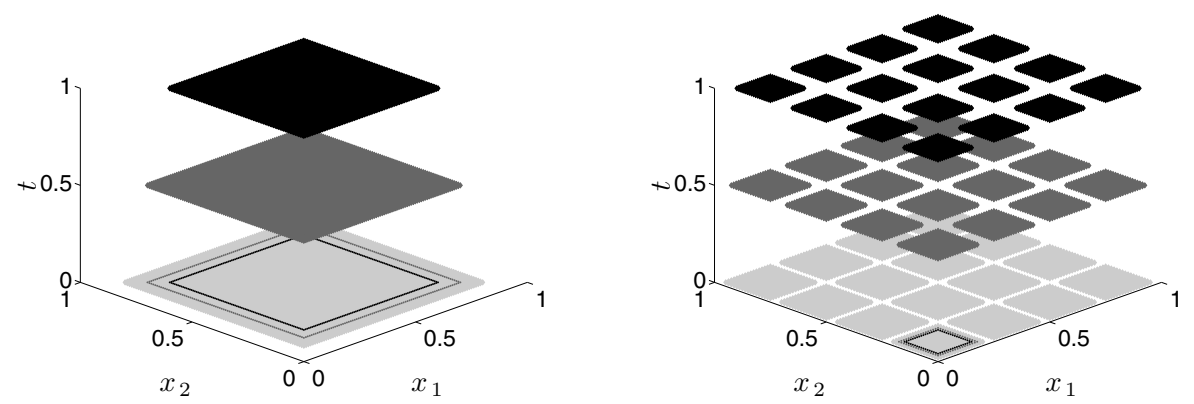

FIG. 10. The images of $\psi_{x}^{\mathrm{w}}$ (left) and $\psi_{\varepsilon}^{\mathrm{w}}$ (right, $\varepsilon=2^{-2}$ ) for different times together with the contour plots of the inner boundary of the cell centered at $\varepsilon(1 / 2,1 / 2)$.

$$
a_{x}(t)=b_{x}(t)=0.1(t+1) .
$$

The transformation $\psi_{x}^{\mathrm{a}}$ can be defined analogously. Since it is actually the "hole" which shrinks in this case, the transformation is slightly more complicated. For ease of notation, we define

$$
\begin{aligned}
& \alpha_{x}\left(x_{1}, t\right)=\left|1 / 2-x_{1}\right|\left(1 / 2-x_{1}\right) \frac{a_{x}(t)-a_{x}(0)}{\left(1 / 2-a_{x}(0)\right)^{2} a_{x}(0)}, \\
& \beta_{x}\left(x_{2}, t\right)=\left|1 / 2-x_{2}\right|\left(1 / 2-x_{2}\right) \frac{b_{x}(t)-b_{x}(0)}{\left(1 / 2-b_{x}(0)\right)^{2} b_{x}(0)} .
\end{aligned}
$$

Then, the analogous transformation is given by

$$
\begin{aligned}
& \left(\tilde{x}_{1}, \tilde{x}_{2}\right)=\left(\psi_{x, 1}^{\mathrm{a}}\left(x_{1}, x_{2}\right), \psi_{x, 2}^{\mathrm{a}}\left(x_{1}, x_{2}\right)\right) \\
& = \begin{cases}\left(x_{1}+\alpha_{x}\left(x_{1}, t\right) x_{2}, x_{2}+\beta_{x}\left(x_{2}, t\right) x_{2}\right), & x_{1} \geq x_{2}, x_{1} \leq 1-x_{2} \\
\left(x_{1}+\alpha_{x}\left(x_{1}, t\right)\left(1-x_{1}\right), x_{2}+\beta_{x}\left(x_{2}, t\right)\left(1-x_{1}\right)\right), & x_{1} \geq x_{2}, x_{1} \geq 1-x_{2} \\
\left(x_{1}+\alpha_{x}\left(x_{1}, t\right)\left(1-x_{2}\right), x_{2}+\beta_{x}\left(x_{2}, t\right)\left(1-x_{2}\right)\right), & x_{1} \leq x_{2}, x_{1} \geq 1-x_{2} \\
\left(x_{1}+\alpha_{x}\left(x_{1}, t\right) x_{1}, x_{2}+\beta_{x}\left(x_{2}, t\right) x_{1}\right), & x_{1} \leq x_{2}, x_{1} \leq 1-x_{2}\end{cases}
\end{aligned}
$$

Again, this can be easily adapted to work out the corresponding transformation $\psi_{\varepsilon}^{\mathrm{a}}$,

$$
\psi_{\varepsilon}^{\mathrm{a}}\left(x_{1}, x_{2}\right)=\varepsilon\left(\left[x_{1} / \varepsilon\right]_{Y}+\psi_{x, 1}^{\mathrm{a}}\left(\{x / \varepsilon\}_{Y}\right),\left[x_{2} / \varepsilon\right]_{Y}+\psi_{x, 2}^{\mathrm{a}}\left(\{x / \varepsilon\}_{Y}\right)\right) .
$$

The functioning of this mapping is illustrated in Figure 11 for the same choice of $a_{x}$ and $b_{x}$ as in Figure 10 (also cf. (5.10)).

For the illustration of the influence of the evolution of the microstructure on the model output, we take a similar setting as in the previous example (interfacial exchange in section 5.1) but allow for an evolution of the domains $\Omega_{\varepsilon}^{\mathrm{a}}$ and $\Omega_{\varepsilon}^{\mathrm{w}}$. Moreover, we choose an intermediate diffusivity for $u^{\text {a }}$, namely $D^{1}=10^{-2} \mathrm{~cm}^{2} / \mathrm{d}$. This implies the choice $k=4 / 9$, and the diffusion term associated with $u^{\text {a }}$ vanishes in the limit. This allows us to focus on the diffusion in the cells and on the interfacial exchange. The initial values are chosen as $u^{\mathrm{a}}(x, 0)=0$ and $u^{\mathrm{w}}(x, y, 0)=\left|Z^{\mathrm{w}}\right|^{-1} \mathrm{e}^{-10\left\|x-(1 / 2,1 / 2)^{T}\right\|^{2}}$ (also cf. Figures 12(left) and 13(left)).

From section 4.1, we find that the limit problem is of similar structure as (5.3) and reads as 

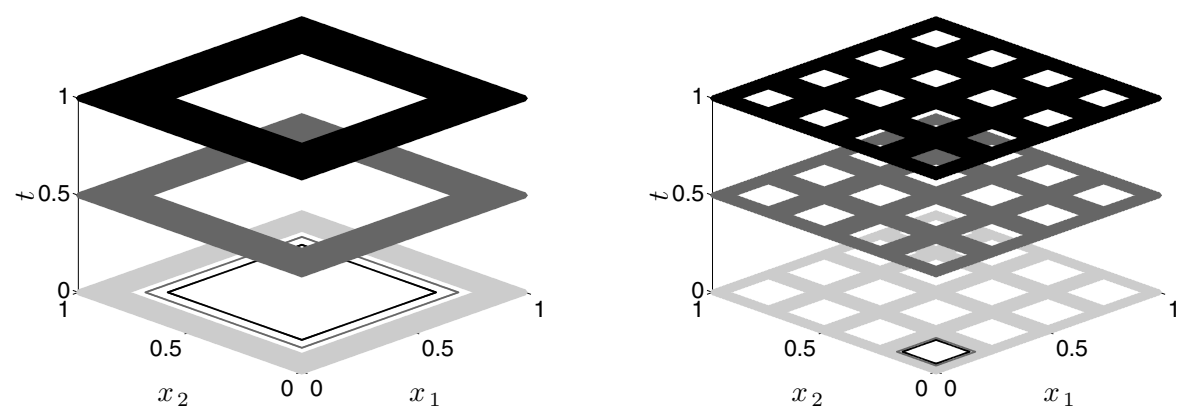

FIG. 11. The images of $\psi_{x}^{\mathrm{a}}$ (left) and $\psi_{\varepsilon}^{\mathrm{a}}$ (right, $\varepsilon=2^{-2}$ ) for different times together with the contour plots of the inner boundary of the cell centered at $\varepsilon(1 / 2,1 / 2)$.

$$
\partial_{t}\left(\int_{Z^{\mathrm{a}}} J^{\mathrm{a}} \mathrm{d} y u^{\mathrm{a}}(x, t)\right)=-\int_{\Gamma}\left\|\Psi^{\mathrm{a}-T} N\right\| J^{\mathrm{a}}\left(u^{\mathrm{a}}-u^{\mathrm{w}}\right) \mathrm{d} y, \quad x \in \Omega, t \in(0,1),
$$

$\partial_{t}\left(J^{\mathrm{w}} u^{\mathrm{w}}(x, y, t)\right)-0.1 \nabla_{y} \cdot\left(J^{\mathrm{w}} \Psi^{\mathrm{w}-1} \Psi^{\mathrm{w}-T} \nabla_{y} u^{\mathrm{w}}\right)=0, \quad x \in \Omega, y \in Z^{\mathrm{w}}, t \in(0,1)$,

$$
-0.1 J^{\mathrm{w}} \Psi^{\mathrm{w}-1} \Psi^{\mathrm{w}-T} \nabla_{y} u^{\mathrm{w}} \cdot \nu^{\mathrm{w}}=-\left\|\Psi^{\mathrm{w}-T} N\right\| J^{\mathrm{w}}\left(u^{\mathrm{a}}-u^{\mathrm{w}}\right), \quad y \in \Gamma, t \in(0,1) .
$$

Recall that the transformations $\psi_{\varepsilon}^{\mathrm{a}}$ and $\psi_{\varepsilon}^{\mathrm{w}}$ are given by (5.13) and (5.8), respectively. It is easily calculated that we have

$$
\Psi^{\mathrm{a}}=\left\{\begin{array}{lc}
{\left[\begin{array}{cc}
1+y_{2} \partial_{y_{1}} \alpha_{x} & 0 \\
\alpha_{x} & 1+y_{2} \partial_{y_{2}} \beta_{x}+\beta_{x}
\end{array}\right],} & y_{1}>y_{2}, y_{1}<1-y_{2}, \\
{\left[\begin{array}{cc}
1+\left(1-y_{1}\right) \partial_{y_{1}} \alpha_{x}-\alpha_{x} & -\beta_{x} \\
0 & 1+\left(1-y_{1}\right) \partial_{y_{2}} \beta_{x}
\end{array}\right],} & y_{1}>y_{2}, y_{1}>1-y_{2}, \\
{\left[\begin{array}{cc}
1+\left(1-y_{2}\right) \partial_{y_{1}} \alpha_{x} & 0 \\
-\alpha_{x} & 1+\left(1-y_{2}\right) \partial_{y_{2}} \beta_{x}-\beta_{x}
\end{array}\right],} & y_{1}<y_{2}, y_{1}>1-y_{2}, \\
{\left[\begin{array}{cc}
1+y_{1} \partial_{y_{1}} \alpha_{x}+\alpha_{x} & \beta_{x} \\
0 & 1+y_{1} \partial_{y_{2}} \beta_{x}
\end{array}\right],} & y_{1}<y_{2}, y_{1}<1-y_{2},
\end{array}\right.
$$

from which $\Psi^{\mathrm{a}-1}, \Psi^{\mathrm{a}-T}$, and $J^{\mathrm{a}}$ can be computed. In particular, we obtain

$$
\begin{aligned}
& J^{\mathrm{a}}= \begin{cases}\left(1+y_{2} \partial_{y_{1}} \alpha_{x}\right)\left(1+y_{2} \partial_{y_{2}} \beta_{x}+\beta_{x}\right), & y_{1}>y_{2}, y_{1}<1-y_{2}, \\
\left(1+\left(1-y_{1}\right) \partial_{y_{1}} \alpha_{x}-\alpha_{x}\right)\left(1+\left(1-y_{1}\right) \partial_{y_{2}} \beta_{x}\right), & y_{1}>y_{2}, y_{1}>1-y_{2}, \\
\left(1+\left(1-y_{2}\right) \partial_{y_{1}} \alpha_{x}\right)\left(1+\left(1-y_{2}\right) \partial_{y_{2}} \beta_{x}-\beta_{x}\right), & y_{1}<y_{2}, y_{1}>1-y_{2}, \\
\left(1+y_{1} \partial_{y_{1}} \alpha_{x}+\alpha_{x}\right)\left(1+y_{1} \partial_{y_{2}} \beta_{x}\right), & y_{1}<y_{2}, y_{1}<1-y_{2},\end{cases} \\
& J_{x}^{\mathrm{a}}\left\|\Psi^{\mathrm{a}-T} N\right\|= \begin{cases}\left(\alpha_{x}^{2}+\left(1+y_{2} \partial_{y_{1}} \alpha_{x}\right)^{2}\right)^{1 / 2}, & y_{1}>y_{2}, y_{1}<1-y_{2}, \\
\left(\left(1+\left(1-y_{1}\right) \partial_{y_{2}} \beta_{x}\right)^{2}+\beta_{x}^{2}\right)^{1 / 2}, & y_{1}>y_{2}, y_{1}>1-y_{2}, \\
\left(\alpha_{x}^{2}+\left(1+\left(1-y_{2}\right) \partial_{y_{1}} \alpha_{x}\right)^{2}\right)^{1 / 2}, & y_{1}<y_{2}, y_{1}>1-y_{2}, \\
\left(\left(1+y_{1} \partial_{y_{2}} \beta_{x}\right)^{2}+\beta_{x}^{2}\right)^{1 / 2}, & y_{1}<y_{2}, y_{1}<1-y_{2} .\end{cases}
\end{aligned}
$$

Copyright (c) by SIAM. Unauthorized reproduction of this article is prohibited. 

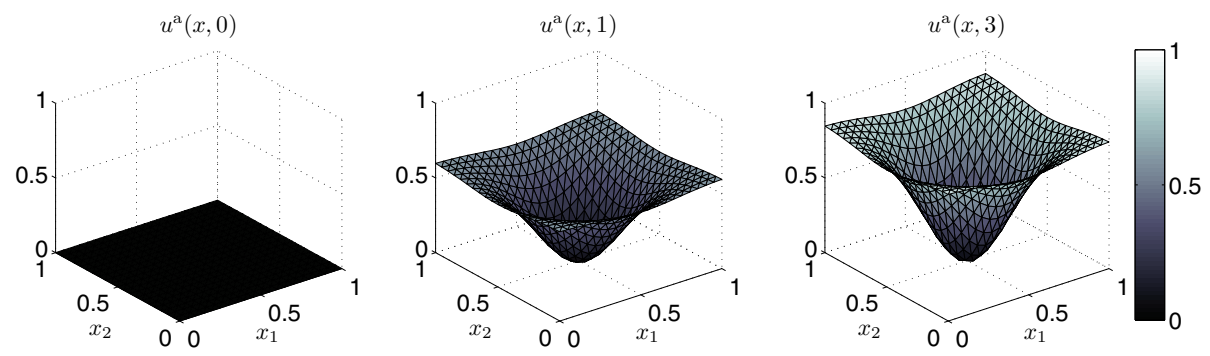

Fig. 12. Profiles of $u^{\mathrm{a}}$ in problem (5.15) for $t=0$ (left), $t=1$ (middle), and $t=3$ (right).

For $Z^{\mathrm{w}}$, the calculation is less technical; namely, we have

$$
\Psi^{\mathrm{w}}=\left[\begin{array}{cc}
\frac{a_{x}(t)-1 / 2}{a_{x}(0)-1 / 2} & 0 \\
0 & \frac{b_{x}(t)-1 / 2}{b_{x}(0)-1 / 2}
\end{array}\right], \quad \Psi^{\mathrm{w}-1}=\Psi^{\mathrm{w}-T}=\left[\begin{array}{cc}
\frac{a_{x}(0)-1 / 2}{a_{x}(t)-1 / 2} & 0 \\
0 & \frac{b_{x}(0)-1 / 2}{b_{x}(t)-1 / 2}
\end{array}\right]
$$

and $J^{\mathrm{w}}=\frac{a_{x}(t)-1 / 2}{a_{x}(0)-1 / 2} \frac{b_{x}(t)-1 / 2}{b_{x}(0)-1 / 2}$.

For simplicity, we assume $b_{x}(t) \equiv a_{x}(t)$; i.e., we assume a square pore-water geometry. In particular, this implies $J^{\mathrm{w}} \Psi^{\mathrm{w}-1} \Psi^{\mathrm{w}-T}=\mathrm{Id}_{2 \times 2}$. Note that this is always the case (in two space dimensions) when the evolution of the pore geometry is given by a simple scaling, i.e., $Z^{\mathrm{w}}(t)=c(t) Z^{\mathrm{w}}(0)$ for some positive function $c(t)$.

We choose $a_{x}(t)=a(t)=0.1(t+1)$ as in Figures 10 and 11. This implies

$$
\alpha_{x}\left(y_{1}, t\right)=\alpha\left(y_{1}, t\right)=\left|1 / 2-y_{1}\right|\left(1 / 2-y_{1}\right) \frac{25 t}{4}, \quad \partial_{y_{1}} \alpha\left(y_{1}, t\right)=-\left|1 / 2-y_{1}\right| \frac{25 t}{2},
$$

$$
\beta_{x}\left(y_{2}, t\right)=\beta\left(y_{2}, t\right)=\left|1 / 2-y_{2}\right|\left(1 / 2-y_{2}\right) \frac{25 t}{4}, \quad \partial_{y_{2}} \beta\left(y_{2}, t\right)=-\left|1 / 2-y_{2}\right| \frac{25 t}{2} .
$$

Then, $\int_{Z^{\mathrm{a}}} J^{\mathrm{a}}$ can be calculated explicitly: We have $\int_{Z^{\mathrm{a}}} J^{\mathrm{a}}=4\left(0.09+0.08 t-0.01 t^{2}\right)$. This value can be calculated even more easily using the current configuration $\int_{Z^{\mathrm{a}}} J^{\mathrm{a}}=$ $\int_{Z^{\mathrm{a}}(t)} 1=1-(1-2 a(t))^{2}$, the results of which are the same, of course.

Simulation results are presented in Figures 12-14. In Figure 12, concentration profiles for $u^{\mathrm{a}}$ are plotted. Note that this concentration is with respect to current pore-air volume. It can be seen that $u^{\mathrm{a}}$ increases from its initial value zero toward the initial value of $u^{\mathrm{w}}$ scaled by the volume factor $\left|Z^{\mathrm{w}}(0)\right| /\left|Z^{\mathrm{a}}(t)\right|$. This is due to the fact that the domain $Z^{\mathrm{w}}$ shrinks while the air-filled pore part increases in volume. Therefore, without interfacial exchange, the real concentration $u^{\mathrm{a}}$ decreases while $u^{\mathrm{w}}$ increases. This accelerates the interfacial exchange, which causes $u^{\mathrm{w}}$ to convert to $u^{\mathrm{a}}$. Metaphorically speaking, the shrinking of pore-water volume causes $u^{\mathrm{w}}$ to be pushed into the pore air. It can be seen in Figures 13 and 14 how the concentration first decreases due to the loss by interfacial exchange and then increases again as the pore-water volume shrinks more quickly (the change of volume is quadratic in time). Thus, the influence of the evolution of the microstructure is well captured by this limit model.

6. Simulation of concrete carbonation. As was discussed in section 1.2, the theoretical investigations of the previous sections were particularly inspired by the real-world problem of concrete carbonation. In this section, we apply the results to 

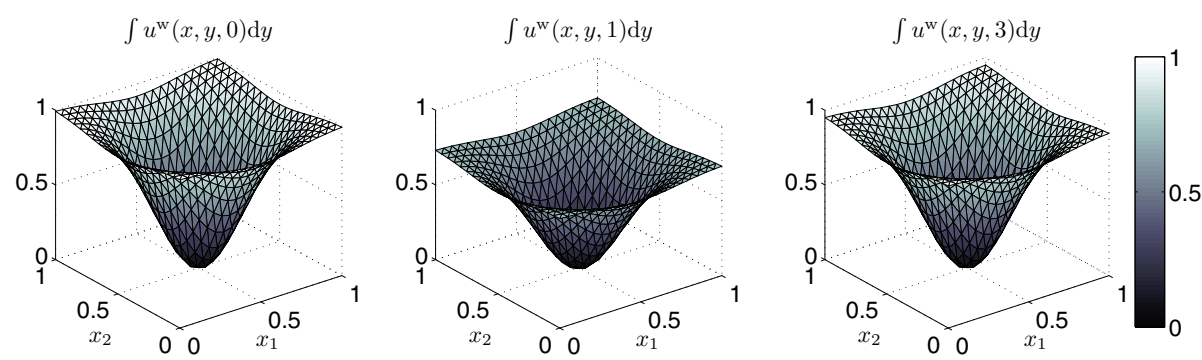

Fig. 13. Profiles of $u^{\mathrm{w}}$ in problem (5.15) for $t=0$ (left), $t=1$ (middle), and $t=3$ (right).
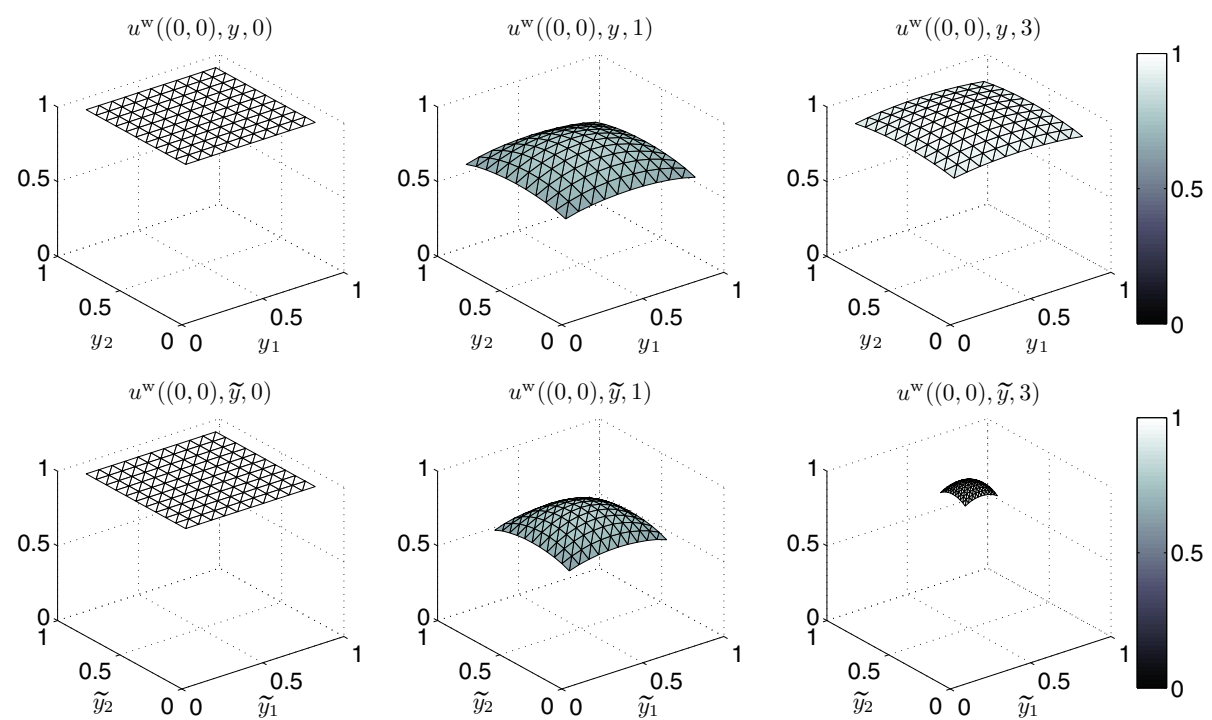

Fig. 14. Cell solutions to (5.15) of the cell at $(0,0)$ for $t=0$ (left), $t=1$ (middle), and $t=3$ (right). Top: reference cell; bottom: current cell.

concrete carbonation. There is a vast literature on concrete carbonation; we particularly refer the reader to $[14,5,6]$ for details on the general subject.

A great number of researchers have tried to find simple formulas for the advancement of the carbonation in a concrete sample (cf. [34, 20] for discussions of these). There are also some works with respect to modelling carbonation with reactiondiffusion systems, most notably $[31,35,18,30,19]$. In the homogenization context, it is also worth noting the article [32], where ion diffusion in concrete is modelled using spatial averaging, and [17], where a two-scale model for a simple carbonation scenario is proposed.

In order to be able to compare the simulation results to experimental data, we restrict our considerations to a particular type of concrete and specific exposure conditions. We assume a piece of concrete based on ordinary Portland cement (OPC) to be exposed to natural atmospheric conditions and compare the simulations with experimental data. To fix ideas, we use the experimental data of [36] for PZ1 concrete with a water-to-cement ratio of 0.6 exposed "out of doors under roof."

The typical capillary pore diameter in OPC-based concrete can vary between $10^{-7} \mathrm{~cm}$ and $10^{-4} \mathrm{~cm} \mathrm{[23].} \mathrm{For} \mathrm{the} \mathrm{present} \mathrm{simulations,} \mathrm{we} \mathrm{take} \ell=10^{-4} \mathrm{~cm}, \ell_{1}^{\mathrm{D}}=1$, and $\ell_{2}^{\mathrm{D}}=\ell_{3}^{\mathrm{D}}=\ell / \sqrt{625}$ as the characteristic microscopic lengths. Note that $\ell_{2}^{\mathrm{D}}$ and 
$\ell_{3}^{\mathrm{D}}$ are essentially smaller than $\ell$ owing to the thinness of the water film in concrete pores. Moreover, we choose $L=1 \mathrm{~cm}$. This implies $\varepsilon=10^{-4}$.

The values of the remaining model parameters refer to an L-shaped cross-section of an OPC concrete sample with a side length of $5 \mathrm{~cm}$ under natural exposure conditions. Our simulations cover a period of 16 years and begin shortly after the curing time. After curing, we assume no $\mathrm{CO}_{2}$ inside the concrete sample and that $0.693 \mathrm{~g} / \mathrm{cm}^{3}$ of $\mathrm{Ca}(\mathrm{OH})_{2}$ is available to the carbonation reaction. In order to account for the fact that carbonation has just started, we assume that the boundary of the concrete sample has already been carbonated. We assume an ambient $\mathrm{CO}_{2}(g)$-concentration resembling natural atmospheric conditions, $u_{1}^{\mathrm{ext}}=0.54 \cdot 10^{-6} \mathrm{~g} / \mathrm{cm}^{3}$.

The diffusion coefficient of $\mathrm{CO}_{2}(\mathrm{~g})$ is $D^{1}=13.8 \cdot 10^{3} \mathrm{~cm}^{2} / \mathrm{d}$, and diffusivities of the ions in water are $D^{2}=D^{3}=1 \mathrm{~cm}^{2} / \mathrm{d}$ [15]. For the interfacial-exchange coefficients at the internal gas-liquid interface and the external boundary, $C^{\text {ex }}$ and $C^{\text {ext }}$, respectively, we take the empirical values $10^{3} / \mathrm{d}$ and the Henry constant is $C^{\mathrm{H}}=0.81$. For the reaction rate, we take $p=q=1$ and use the empirical values $r^{i}=m_{i} 500 \mathrm{~mol} \cdot \mathrm{cm}^{3} /\left(\mathrm{g}^{2} \mathrm{~d}\right), i=\mathrm{u}, \mathrm{v}$, where $m_{i}$ are the molar weights; we have $m_{\mathrm{u}}=m_{\mathrm{A}}=44 \mathrm{~g} / \mathrm{mol}$ and $m_{\mathrm{v}}=m_{\mathrm{B}}=74 \mathrm{~g} / \mathrm{mol}$.

Taking $u^{\mathrm{a}}=\left[\mathrm{CO}_{2}(g)\right], u^{\mathrm{w}}=\left[\mathrm{CO}_{2}(a q)\right]$, and $v^{\mathrm{w}}=\left[\mathrm{Ca}(\mathrm{OH})_{2}(a q)\right]$, the microscopic problem without exchange with the environment reads exactly as (1.4).

The data given above yield characteristic times of

$$
T^{\mathrm{a}}=10^{-4 k} 1^{2-k} / 13.8 \cdot 10^{3}, \quad T^{\mathrm{w}}=25^{l} 10^{-4 l} 1^{2-l}, \quad T_{\mathrm{v}}^{\mathrm{w}}=25^{j} 10^{-4 j} 1^{2-j} .
$$

According to section 2.1, this implies $k=0, l=j \approx 1.59$, and $T^{\mathrm{a}}=T^{\mathrm{w}}=T_{\mathrm{v}}^{\mathrm{w}}=$ $1 / 13.8 \cdot 10^{3}$. For the scaling exponent of the interfacial-exchange term, we obtain $m \approx 0.29$.

Therefore (cf. section 4.1), the homogenized limit problem is of purely macroscopic type without diffusion of $u^{\mathrm{w}}$ and $v^{\mathrm{w}}$ (formally, this corresponds to $P^{\mathrm{w}}=0$ ) and we have no interfacial-exchange term. Instead, we obtain $C^{\mathrm{H}} u^{\mathrm{a}}=u^{\mathrm{w}}$ in the limit. Altogether, the homogenized limit model in dimensional form is given by

$$
\begin{aligned}
& \partial_{t}\left(\left(J^{\mathrm{a}}\left|Z^{\mathrm{a}}\right|+J^{\mathrm{w}}\left|Z^{\mathrm{w}}\right|\right) u(x, t)\right)-\nabla \cdot\left(P^{\mathrm{a}} \nabla u\right)=-J^{\mathrm{w}}\left|Z^{\mathrm{w}}\right| r^{\mathrm{u}} u v^{\mathrm{w}}, \quad x \in \Omega, t \in S, \\
& \left|Z^{\mathrm{w}}\right| \partial_{t}\left(J^{\mathrm{w}} v^{\mathrm{w}}(x, t)\right)=-J^{\mathrm{w}}\left|Z^{\mathrm{w}}\right| C^{\mathrm{H}} r^{\mathrm{v}} u v^{\mathrm{w}}, \quad x \in \Omega, t \in S, \\
& -P^{\mathrm{a}} \nabla u \cdot \nu=C^{\mathrm{ext}}\left(u-u^{\mathrm{ext}}\right), \quad x \in \partial \Omega, t \in S,
\end{aligned}
$$

where we have added a Robin boundary condition for $\mathrm{CO}_{2}(g)$ to account for the exchange with the environment. We take the empirical value $C^{\text {ext }}=10^{3} / \mathrm{d}$, and $u_{1}^{\text {ext }}$ is given by the ambient $\mathrm{CO}_{2}$ concentration (see above). Moreover, $u$ now represents a combined $\mathrm{CO}_{2}$ concentration referring to both pore air and pore water.

It can be noted that $(6.2 \mathrm{a})$ and $(6.2 \mathrm{~b})$ can even be combined to one equation by solving (6.2b) for $v^{\mathrm{w}}$ and substitution of the result into (6.2a). However, we leave the system as it is since we are also particularly interested in the concentration profiles of $v^{\mathrm{w}}$. Also note that the concentration $u$ now refers to the complete pore volume. Moreover, the fast exchange at the exterior boundary could also be well approximated by a Dirichlet condition, i.e., $u=u^{\text {ext }}$ on $\partial \Omega$.

Before solving the system of equations (6.2), we must specify the functions related to the evolution of the subdomains, i.e., $J^{\mathrm{a}}, \Psi^{\mathrm{a}}$, and $J^{\mathrm{w}}$ ( $\Psi^{\mathrm{a}}$ is contained in the definition of $P^{\mathrm{a}}$; cf. (4.2)). For this, recall that $\Psi^{i}$ relates the length and orientation 
of a material fibre in the reference configuration to its length and orientation in the current configuration and $J^{i}$ describes the change of volume, $i \in\{\mathrm{a}, \mathrm{w}\}$. Since water can be assumed incompressible, it is reasonable to set $J^{\mathrm{w}}=1$. Diffusion of $\mathrm{CO}_{2}$ in air is fast; hence, we assume that the influence of the microstructure on the direction of the flux is negligible; i.e., $\Psi^{\mathrm{a}} \approx 1$ in the definition of (4.2). We are left with deriving an equation for $J^{\text {a }}$, which must be coupled to the reaction since the change of volume of pore air is due to the conversion of $\mathrm{Ca}(\mathrm{OH})_{2}$ to $\mathrm{CaCO}_{3}$.

The dissolution of $\mathrm{Ca}(\mathrm{OH})_{2}$ and precipitation of $\mathrm{CaCO}_{3}$ are fast, which is why we approximate them as being instantaneous. In the cell $Y$ at the point $x$, the amount (i.e., mass) of $\mathrm{Ca}(\mathrm{OH})_{2}$ being used up and of $\mathrm{CaCO}_{3}$ being produced at time $t$ is given by

$$
\int_{Z^{\mathrm{w}}} m_{\mathrm{B}} J^{\mathrm{w}} f^{\mathrm{r}} \mathrm{d} y \text { and } \int_{Z^{\mathrm{w}}} m_{\mathrm{C}} J^{\mathrm{w}} f^{\mathrm{r}} \mathrm{d} y
$$

respectively. Noting that $J^{\mathrm{w}}=1$ and that an increase of total volume of constituents implies a decrease of volume of pore air, we have

$$
\frac{\mathrm{d}}{\mathrm{d} t} \int_{Z^{\mathrm{a}}} J^{\mathrm{a}} \mathrm{d} y=-\left|Z^{\mathrm{a}}\right| C^{\mathrm{m}} \int_{Z^{\mathrm{w}}} f^{\mathrm{r}} \mathrm{d} y, \quad \text { where } \quad C^{\mathrm{m}}=\frac{1}{\left|Z^{\mathrm{a}}\right|}\left(\frac{m_{\mathrm{C}}}{\rho_{\mathrm{C}}}-\frac{m_{\mathrm{B}}}{\rho_{\mathrm{B}}}\right)
$$

for each instant in time, where $\rho_{\mathrm{B}}$ and $\rho_{\mathrm{C}}$ are the densities of $\mathrm{Ca}(\mathrm{OH})_{2}$ and $\mathrm{CaCO}_{3}$, respectively.

Since we do not expect $J^{\text {a }}$ to vary within one cell (and since we do not have any more information), we define $J^{\mathrm{a}}(x, t)$ to be the constant value determined by (6.4) in each cell. Hence, an equation for $J^{\mathrm{a}}$ is obtained: Find $J^{\mathrm{a}}: \Omega \times S \rightarrow \mathbb{R}$ such that

$$
\partial_{t} J^{\mathrm{a}}(x, t)=-\left|Z^{\mathrm{w}}\right| C^{\mathrm{m}} r_{\mathrm{J}} u^{\mathrm{w}} v^{\mathrm{w}}, \quad J^{\mathrm{a}}(0) \equiv 1,
$$

where $r_{\mathrm{J}}=500 \mathrm{~mol} \cdot \mathrm{cm}^{3} /\left(\mathrm{g}^{2} \mathrm{~d}\right)$ as before. Using the data from [15], we have $\left|Z^{\mathrm{a}}\right| C^{\mathrm{m}}=$ $(100 / 2.71-74 / 2.2)=3.26$. The volume fractions are $\left|Z^{\mathrm{a}}\right|=0.18$ and $\left|Z^{\mathrm{w}}\right|=0.1$ [23]. Note that no assumption on the specific pore geometry has been made in the derivation of $(6.5)$.

We do not calculate the macroscopic diffusion tensor but use empirical value $Q^{\mathrm{a}}=2.2 \cdot 10^{-3}$ instead $[23,35]$.

Making use of the equality $C^{\mathrm{H}} u^{\mathrm{a}}=u^{\mathrm{w}},(6.5)$ can be written as

$$
\partial_{t} J^{\mathrm{a}}(x, t)=-\left|Z^{\mathrm{w}}\right| C^{\mathrm{m}} r_{\mathrm{J}} C^{\mathrm{H}} u v^{\mathrm{w}} .
$$

The rest of the limit problem simplifies to

$$
\begin{aligned}
\partial_{t}\left(\left(J^{\mathrm{a}}\left|Z^{\mathrm{a}}\right|+\left|Z^{\mathrm{w}}\right|\right) u(x, t)\right)-\nabla \cdot\left(J^{\mathrm{a}} Q^{\mathrm{a}} \nabla u\right) & =-\left|Z^{\mathrm{w}}\right| r^{\mathrm{u}} u v^{\mathrm{w}}, & & x \in \Omega, t \in S, \\
\left|Z^{\mathrm{w}}\right| \partial_{t} v^{\mathrm{w}}(x, t) & =-\left|Z^{\mathrm{w}}\right| C^{\mathrm{H}} r^{\mathrm{v}} u v^{\mathrm{w}}, & & x \in \Omega, t \in S, \\
-J^{\mathrm{a}} P^{\mathrm{a}} \nabla u \cdot \nu & =C^{\mathrm{ext}}\left(u-u^{\text {ext }}\right), & & x \in \partial \Omega, t \in S .
\end{aligned}
$$

This system is considerably simpler than the original microscopic system. Nevertheless, the correct scaling ensures that all processes are captured accurately in this limit model. The evolution of the microstructure is accounted for by the coupled nature of the equation for the pore-volume factor $J^{\text {a }}(6.6)$ with the reaction-diffusion system (6.6b)-(6.6d). The computational effort to solve this limit model is rather small (of 
the order of minutes on a modern computer), while for the simulation of the microscopic model $3 / 4 \cdot\left(5 \cdot 10^{4}\right)^{2}$ evolving pores of side length $10^{-4} \mathrm{~cm}$ would have been needed to be discretized.

Before presenting simulation results, we want to estimate the effect on the pore-air volume. Initially, we have $\left|Z^{\mathrm{w}}\right| v_{0}^{\mathrm{w}} / m_{\mathrm{B}}=9.36 \cdot 10^{-4} \mathrm{~mol} \mathrm{Ca}(\mathrm{OH})_{2}$ per $\mathrm{cm}^{3}$ of concrete. Therefore, of each $\mathrm{cm}^{3}$ of uncarbonated concrete, $\left|Z^{\mathrm{w}}\right| v_{0}^{\mathrm{w}} / \rho_{\mathrm{B}}=0.0315 \mathrm{~cm}^{3}$ are taken up by $\mathrm{Ca}(\mathrm{OH})_{2}$. After carbonation, each mole of $\mathrm{Ca}(\mathrm{OH})_{2}$ has been converted to a mole of $\mathrm{CaCO}_{3}$; i.e., we have $9.36 \cdot 10^{-4} \mathrm{~mol} \mathrm{CaCO}_{3}$ per $\mathrm{cm}^{3}$ of concrete. This takes up a volume of $m_{\mathrm{C}} / \rho_{\mathrm{C}} 9.36 \cdot 10^{-4} \mathrm{~mol} / \mathrm{cm}^{3}=0.0346$ per $\mathrm{cm}^{3}$ of carbonated concrete. Therefore, $0.0031 \mathrm{~cm}^{3}$ more volume is taken up by the solids per $\mathrm{cm}^{3}$ of concrete after carbonation. This corresponds to a reduction of pore-air volume of approximately $1.7 \%$.

From the literature (cf. $[5,23]$ ), we find that the reduction of pore volume by carbonation is between approximately $10 \%$ and $30 \%$ depending on various parameters, particularly on the type of cement used. The discrepancy between the measured values and the one calculated above is discussed in [5], and several possible reasons are given. They are mostly associated with the fact that the precipitation and formation of solid $\mathrm{CaCO}_{3}$ are more complicated than the very simple assumptions made here so that the density of precipitated $\mathrm{CaCO}_{3}$ in carbonated concrete is actually considerably smaller than it normally is.

We want to take into account this larger change of volume. For the simulations, we take a value of $14.5 \%$. Under the assumption that the pore-water volume remains constant, this corresponds to a reduction of pore-air volume by $21 \%$.

The solution to (6.6) is plotted in Figure 15. Dimensional quantities are shown except for the concentrations which we plot in a normalized way, that is, $u / u^{\text {ext }}$ and $v^{\mathrm{w}} / v_{0}^{\mathrm{w}}$. It can be observed that the bulk of the carbonation reaction is concentrated on a narrow zone which advances into the concrete with time, and the $\mathrm{Ca}(\mathrm{OH})_{2}{ }^{-}$ concentration exhibits steep gradients near the reaction zone. This coincides with other simulation results; cf. [23, 18].

An important quantity for durability issues is the carbonation depth, i.e., the depth the carbonation zone has penetrated into the concrete sample after a given amount of time. Similarly to [35], we define the carbonation depth as the level set $v^{\mathrm{w}} / v_{0}^{\mathrm{w}}=0.1$. In Figure 16(right), the predicted carbonation depth is plotted in comparison to experimental data by [36] (long-term exposure out of doors under roof). It can be seen that good approximation of experimental data is achieved. In particular, an overestimation of the carbonation depth toward the end of the considerations, which usually results when neglecting the evolution of the microstructure [20, 18], does not occur here.

7. Summary. A prototypical reaction-diffusion system was considered modelling the evolution of concentrations in a porous medium with evolving microstructure. A nondimensionalization was performed taking into account the different characteristic lengths and times. Macroscopic limit models were then obtained from these scaled micromodels making use of the method of homogenization in domains with evolving microstructure. Numerical experiments validated the appropriateness of the scaling depending on the material parameters as well as accounting for the evolution of the microstructure. The general considerations were then applied to the specific real-world problem concrete carbonation. It turned out that a comparably simple macroscopic model arises in the homogenization limit, which compares well to experimental data. 

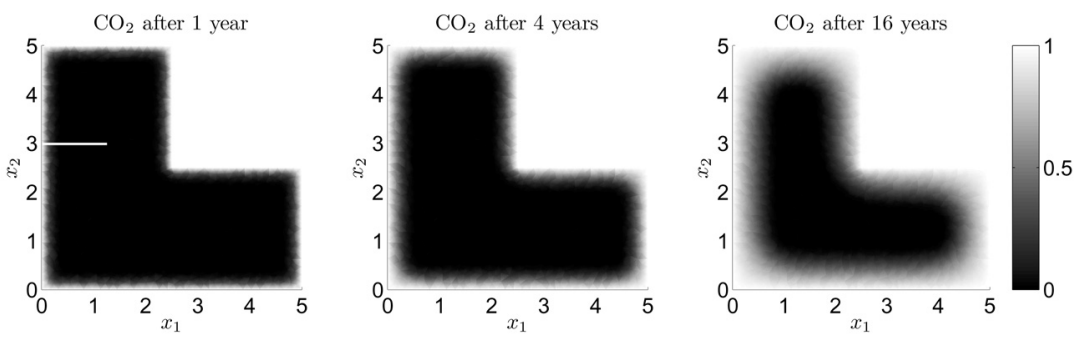

$\mathrm{Ca}(\mathrm{OH})_{2}$ after 1 year

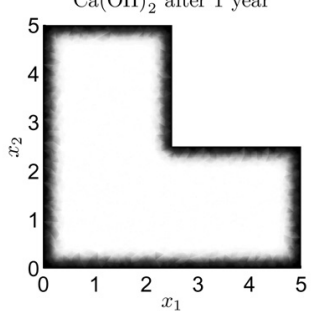

$\mathrm{Ca}(\mathrm{OH})_{2}$ after 4 years

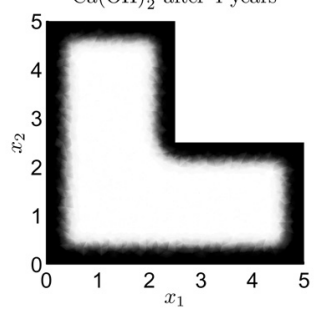

$\mathrm{Ca}(\mathrm{OH})_{2}$ after 16 years
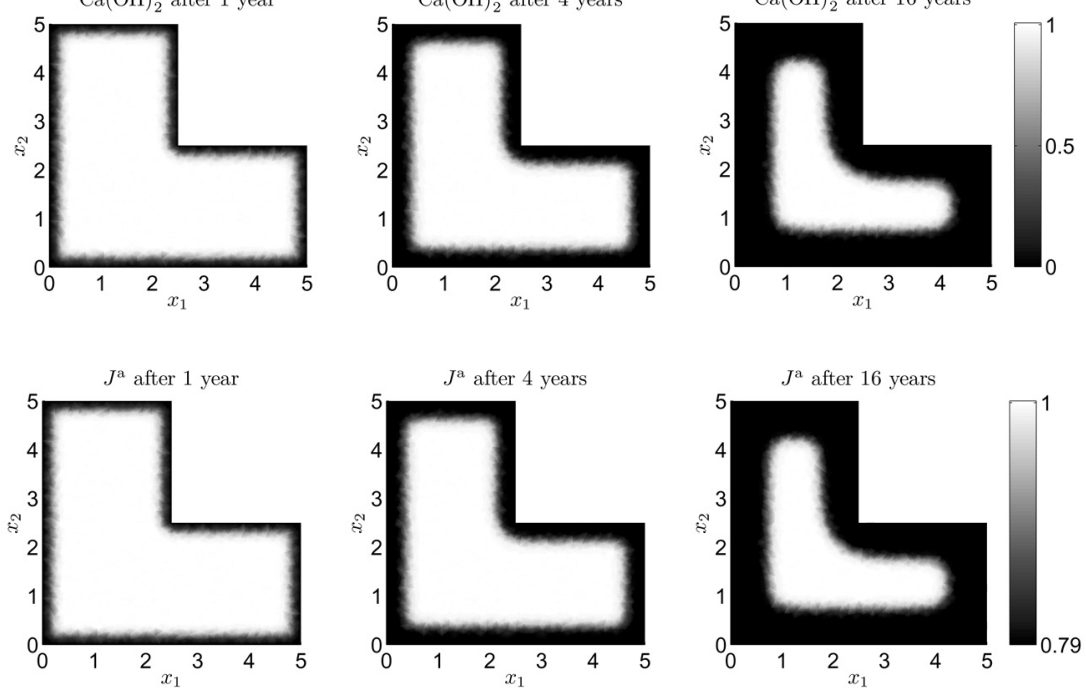

Fig. 15. Solution to problem (6.6). $\mathrm{CO}_{2}$ (top) and $\mathrm{Ca}(\mathrm{OH})_{2}$ (middle) concentrations as well as $J^{\mathrm{a}}$ (bottom) after 1 year (left), 4 years (middle), and 16 years (right). In the top left plot, the cut is depicted for which the solutions are presented in Figure 16.
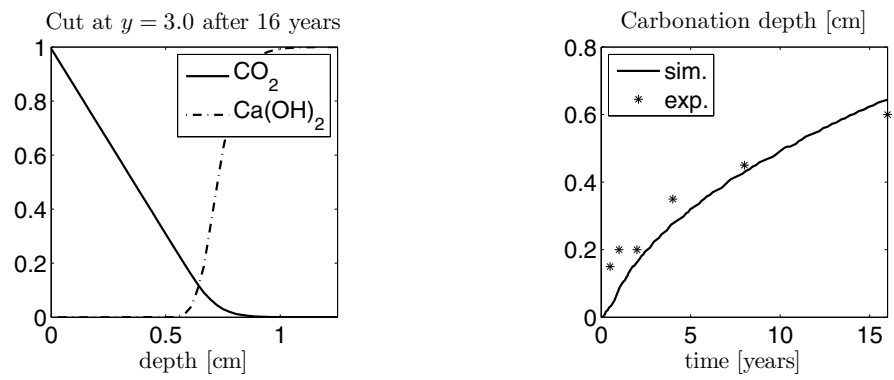

FIG. 16. Results for the carbonation model with evolving microstructure. Left: concentration profiles at the cut at $x_{2}=3$ (depicted in Figure 15). Right: carbonation depth versus time compared to experimental data by [36].

Acknowledgments. Fruitful discussions with S. A. Meier from the University of Bremen, A. Muntean from the Technical University of Eindhoven, W. Jäger from the University of Heidelberg, and J. Kropp from the Bremen University of Applied Sciences during this research are greatly appreciated. 


\section{REFERENCES}

[1] H.-D. Alber, Evolving microstructure and homogenization, Contin. Mech. Thermodyn., 12 (2000), pp. 235-286.

[2] G. Allaire, Homogenization and two-scale convergence, SIAM J. Math. Anal., 23 (1992), pp. $1482-1518$.

[3] J. Bear and Y. Bachmat, Introduction to Modeling of Transport Phenomena in Porous Media, Kluwer Academic Publishers, Dordrecht, The Netherlands, 1990.

[4] A. Bensoussan, J.-L. Lions, and G. Papanicolaou, Asymptotic Analysis for Periodic Structures, North-Holland, Amsterdam, New York, 1978.

[5] T. A. Bien, Karbonatisierung und Realkalisierung von Zementstein und Beton, Ph.D. thesis, University of Karlsruhe, Karlsruhe, Germany, 1988.

[6] D. Bunte, Zum karbonatisierungsbedingten Verlust der Dauerhaftigkeit von Aussenbauteilen aus Stahlbeton, Institut für Baustoffe, Massivbau und Brandschutz der Technischen Universität Braunschweig 107, Technische Universität Braunschweig, Institut für Baustoffe, Braunschweig, Germany, 1994.

[7] T. Chaussadent, États de lieux et réflexions sur la carbonatation du beton armé, Technical report LCPC OA29, Laboratoire Central de Ponts et Chaussées, Paris, 1999.

[8] D. Cioranescu and P. Donato, An Introduction to Homogenization, The Clarendon Press, Oxford University Press, New York, 1999.

[9] C. ECK, Analysis of a two-scale phase field model for liquid-solid phase transitions with equiaxed dendritic microstructure, Multiscale Model. Simul., 3 (2004), pp. 28-49.

[10] C. ECK, Homogenization of a phase field model for binary mixtures, Multiscale Model. Simul., 3 (2004), pp. 1-27.

[11] P. Goel, J. Sneyd, And A. Friedman, Homogenization of the cell cytoplasm: The calcium bidomain equations, Multiscale Model. Simul., 5 (2006), pp. 1045-1062.

[12] U. Hornung, ED., Homogenization and Porous Media, Springer, New York, 1997.

[13] U. HoRnung AND W. JäGER, Diffusion, convection, adsorption, and reaction of chemicals in porous media, J. Differential Equations, 92 (1991), pp. 199-225.

[14] J. KRopP, Relations between transport characteristics and durability, in Performance Criteria for Concrete Durability, RILEM Report 12, J. Kropp and H. K. Hilsdorf, eds., E \& FN SPON, London, 1995, pp. 97-137.

[15] D. R. Lide, ED., CRC Handbook of Chemistry and Physics, 82nd ed., CRC Press, Boca Raton, FL, 2001.

[16] J. D. Logan, Transport Modeling in Hydrogeochemical Systems, Springer, New York, 2001.

[17] S. A. Meier, M. A. Peter, And M. Böhm, A two-scale modelling approach to reactiondiffusion processes in porous materials, Comput. Mater. Sci., 39 (2007), pp. 29-34.

[18] S. A. Meier, M. A. Peter, A. Muntean, And M. Böhm, Modelling and simulation of concrete carbonation with internal layers, Chem. Eng. Sci., 62 (2007), pp. 1125-137.

[19] A. Muntean, A Moving-Boundary Problem: Modeling, Analysis and Simulation of Concrete Carbonation, Cuvillier, Göttingen, Germany, 2006.

[20] A. Muntean, S. A. Meier, M. A. Peter, M. Böhm, And J. Kropp, A Note on the Limitations of the Use of Accelerated Concrete-Carbonation Tests for Service-Life Predictions, Berichte aus der Technomathematik 05-04, ZeTeM, University of Bremen, Bremen, Germany, 2005.

[21] M. Panfilov, Macroscale Models of Flow through Highly Heterogeneous Porous Media, Kluwer Academic Publishers, Dordrecht, The Netherlands, 2000.

[22] L. Pankratov, A. Piatnitskit, and V. Rybalko, Homogenized model of reaction-diffusion in a porous medium, C. R. Mécanique, 331 (2003), pp. 253-258.

[23] V. G. Papadakis, C. G. Vayenas, and M. N. Fardis, A reaction engineering approach to the problem of concrete carbonation, AIChE J., 35 (1989), pp. 1639-1650.

[24] M. A. Peter, Coupled Reaction-Diffusion Processes and Evolving Microstructure: Mathematical Modelling and Homogenisation, Logos Verlag, Berlin, 2007.

[25] M. A. Peter, Homogenisation in domains with evolving microstructure, C. R. Mécanique, 335 (2007), pp. 357-362.

[26] M. A. Peter, Homogenisation of a chemical degradation mechanism inducing an evolving microstructure, C. R. Mécanique, 335 (2007), pp. 679-684.

[27] M. A. PETER, Coupled reaction-diffusion processes inducing an evolution of the microstructure: Analysis and homogenization, Nonlinear Anal., 70 (2009), pp. 806-821.

[28] M. A. Peter And M. Böhm, Scalings in homogenisation of reaction, diffusion and interfacial exchange in a two-phase medium, in Proceedings of Equadiff 11, M. Fila, A. Handlovicova, K. Mikula, M. Medved, P. Quittner, and D. Sevcovic, eds., Comenius University Press, Bratislava, Slovakia, 2007, pp. 369-376.

Copyright $@$ by SIAM. Unauthorized reproduction of this article is prohibited. 
[29] M. A. Peter And M. BöHm, Different choices of scaling in homogenization of diffusion and interfacial exchange in a porous medium, Math. Methods Appl. Sci., 31 (2008), pp. 12571282.

[30] M. A. Peter, A. Muntean, S. A. Meier, and M. Böhm, Competition of several carbonation reactions in concrete: A parametric study, Cem. Concr. Res., 38 (2008), pp. 1385-1393.

[31] A. V. Saetta, B. A. Schrefler, and R. V. Vitaliani, The carbonation of concrete and the mechanism of moisture, heat and carbon dioxide flow through porous materials, Cem. Concr. Res., 23 (1993), pp. 761-772.

[32] E. Samson, J. Marchand, and J. J. Beaudoin, Describing ion diffusion mechanisms in cement-based materials using the homogenization technique, Cem. Concr. Res., 29 (1999), pp. 1341-1345.

[33] E. SAnchez-Palencia, Nonhomogeneous Media and Vibration Theory, Springer, Berlin, New York, 1980.

[34] K. Sisomphon, Influence of Pozzolanic Material Additions on the Development of the Alkalinity and the Carbonation Behaviours of Composite Cement Pastes and Concretes, Ph.D. thesis, Technical University Hamburg-Harburg, Hamburg, Germany, 2004.

[35] A. Steffens, D. Dinkler, And H. Ahrens, Modeling carbonation for corrosion risk prediction of concrete structures, Cem. Concr. Res., 32 (2002), pp. 935-941.

[36] H.-J. WiERIG, Longtime studies on the carbonation of concrete under normal outdoor exposure, in Proceedings of the RILEM, Hannover University, Hannover, Germany, 1984, pp. 239249 .

Copyright (C) by SIAM. Unauthorized reproduction of this article is prohibited. 